\title{
Introducing Chirality at Phosphorus Atoms: An Update on the Recent Synthetic Strategies for the Preparation of Optically Pure P-Stereogenic Molecules
}

\author{
Sébastien Lemouzy, ${ }^{*[a]}$ Laurent Giordano, ${ }^{[a]}$ Damien Hérault, ${ }^{[a]}$ and Gérard Buono ${ }^{[a]}$
}

Abstract: The synthesis of phosphorus molecules presenting a chiral center on the P-atom, also known as P-stereogenic compounds, has long attracted the curiosity of the scientific community. Indeed, these chemical compounds feature many peculiar properties, allowing their use in various fields of applications, ranging from medicine to enantioselective catalysis. However, their synthesis, and more particularly the introduction and retention of the chiral information on the phosphorus center, remains a very challenging task. That is why this review article focuses on the recent advances in the enantioselective synthesis of P-stereogenic molecules, with a particular focus on the introduction of the chiral center on the phosphorus atom. This article summarizes the main synthetic approaches directed towards the enantioselective synthesis of such chemical entities with a historical perspective. Thus, approaches based on the use of chiral auxiliaries attached to the phosphorus atom and the use of chiral stoichiometric reagents will be discussed first, as they were historically the first to be developed. Then, the recent discoveries in the catalytic and enantioselective synthesis and the direct optical resolution of P-chiral compounds will be discussed.

\section{Introduction}

The access to chiral phosphorus compounds has always been considered as a challenge in modern organic synthesis. Indeed, phosphorus compounds can be used in a wide array of different application areas, by taking advantage of the two different stable oxidation states $\left(+\mathrm{III}\right.$ and $+\mathrm{V}$ ) of the phosphorus center. ${ }^{[1]}$ Indeed, phosphorus oxides usually display interesting biological properties, and thus they have been applied in medicine (as HIV protease inhibitor ${ }^{[2]}$ or anticancer agents ${ }^{[3]}$ ) as well as crop protection (as herbicides ${ }^{[4]}$ or virucides ${ }^{[5]}$ ). On the other hand, phosphorus(III) compounds can be used as efficient ligands for transition-metal catalysis, ${ }^{[6]}$ as well as organo-catalysts themselves. ${ }^{[7]}$ Therefore, the design of chiral phosphorus(III) and (V) compounds is of utmost importance, especially in the field of asymmetric catalysis.

Among these compounds, those possessing a chiral backbone have attracted the most attention, and thus represent the vast majority of chiral phosphorus ligands and catalysts (Figure 1). In parallel to these chiral phosphorus molecules, the ones featuring the chiral center on the phosphorus atom (also known as P-stereogenic compounds) have seen less develop- ment, mostly due to synthetic reasons. Indeed, unlike most nitrogen compounds, phosphorus compounds feature a configurationally stable chiral center on the heteroatom (Scheme 1). ${ }^{[8]}$
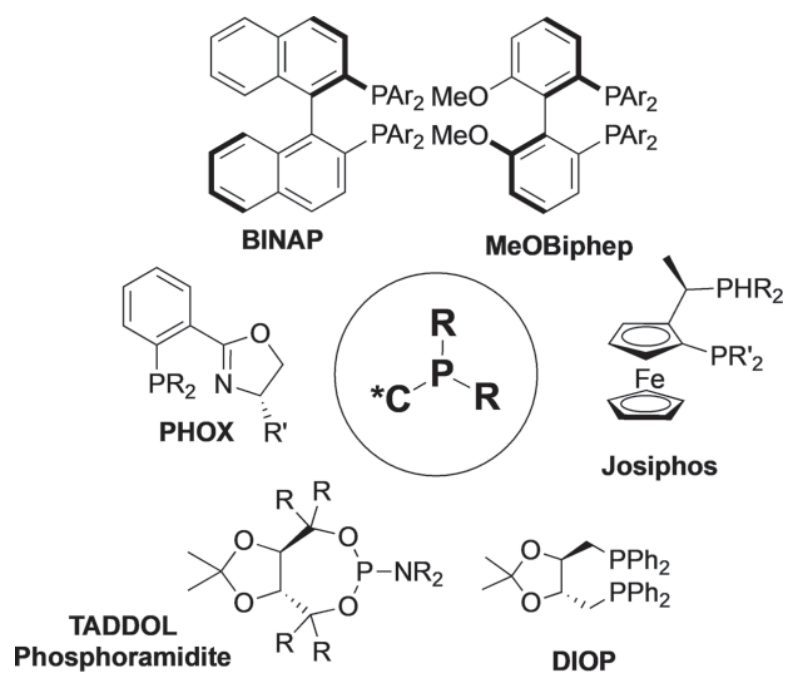

Figure 1. Selected phosphorus ligands attached to a chiral carbon backbone.

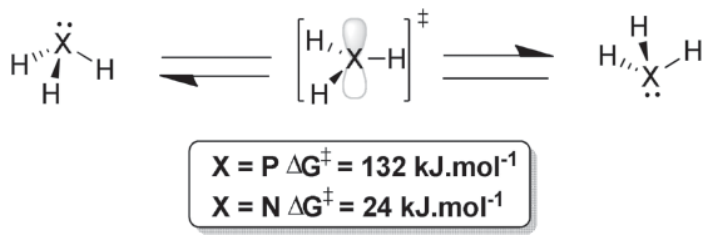

Scheme 1. Configurational stability of phosphines vs. amines. 
These compounds can be used successfully as ligands or organocatalysts in asymmetric catalysis, and they often outperform their chiral backbone substituted counterparts in terms of enantioselectivity. ${ }^{[9]}$
Thus, the synthesis of P-stereogenic molecules is of paramount importance to increase the ligand portfolio in asymmetric catalysis. Since the pioneering work of Meisenheimer and Lichtenstadt, who isolated the first enantioenriched P-stereo-

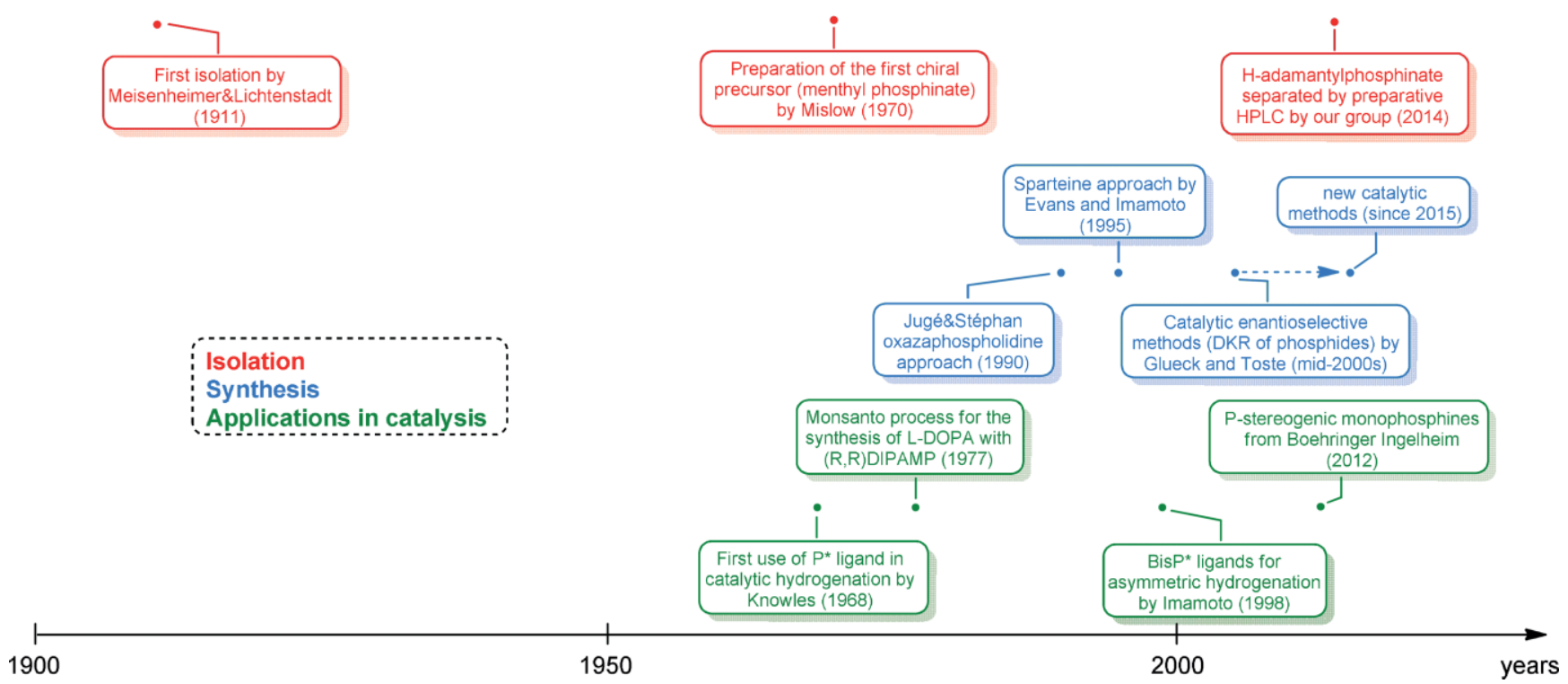

Figure 2. Selected important dates in the preparation and use of P-stereogenic molecules. 
genic compound (ethyl methyl phenylphosphine oxide, via resolution with (+)-bromocamphorsulfonic acid) in 1911, ${ }^{[10]}$ the development of synthetic methods for the preparation of such compounds has acknowledged many discoveries, especially in the last three decades (Figure 2). That is why this review aims to present the recent developments in the synthesis of P-stereogenic molecules. It will cover the following approaches:

- Via a chiral auxiliary bound to the $\mathrm{P}$ atom

- Via a chiral stoichiometric reagent

- Via catalytic enantioselective reactions

- Via semi-preparative chiral separation

- Via the formation of diastereomeric complexes

The synthesis of P-stereogenic compounds by enzymatic catalysis and related biocatalyzed processes is beyond the scope of this review. Likewise, the functionalization of enantiopure P-stereogenic molecules ${ }^{[11]}$ will only be treated superficially, as this review focus mostly on the asymmetric strategies developed for the introduction of the chiral element on the phosphorus atom.

\section{Synthesis of P-Stereogenic Compounds via a Chiral Auxiliary Bound to the Phosphorus Atom}

\section{1. (-)-Menthol}

Historically, the methods using a chiral auxiliary attached to the phosphorus atom were the first to be developed. Indeed, in 1970, the group of Mislow described the first synthesis of diastereomerically pure (-)-menthyl (phenyl) H-phosphinate 1, from cheap and readily available (-)-menthol, through recrystallization. ${ }^{[12]}$

More recently, many research groups including Han group, ${ }^{[13]}$ Montchamp, ${ }^{[14]}$ as well as our group ${ }^{[15]}$ have shown the synthetic potential of this precursor (Scheme 2). Indeed, 1 can be reacted in the presence of organolithium or organomagnesium reagents (via $S_{N} 2$ mechanism on the $\sigma^{3} \lambda^{3}$ deprotonated intermediate) to yield the corresponding secondary phosphine oxides (SPOs) with high enantiospecificity, ${ }_{1}^{[13 b, 15 a]}$ as minor loss of optical activity is usually observed. Our group has shown that these SPOs could be converted to either enantiopure phosphinous acid-boranes ${ }^{[15 b]}$ or secondary phosphine-boranes. ${ }^{[15 c]}$ 1 can also be functionalized in the presence of carbonyl electrophiles ${ }^{[13 c, 14]}$ to generate the corresponding hydroxyalkyl phosphinates with high diastereomeric ratios.

The palladium-catalyzed Markovnikov hydrophosphination of alkynes was also described with (-)-menthyl(phenyl) phosphinate, yielding vinyl phosphinates with good yields and retention of the chiral information on the phosphorus center. ${ }^{[13 a]}$ Recently, another method relying on diastereomerically pure $O$ (-)-menthyl phosphinous acid monoester monoamide-borane 2, was developed by Stankevic, allowed the highly enantioselective preparation of various phosphorus-boranes compounds (Scheme 3). ${ }^{[16]}$

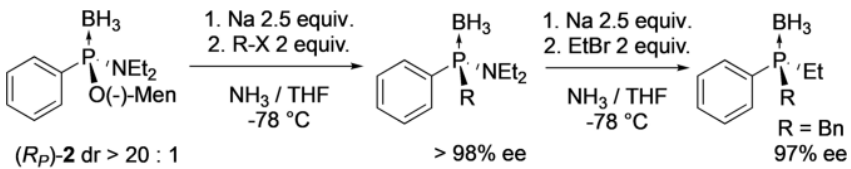

Scheme 3. O-(-)-menthyl phosphinous acid monoester monoamide -boranes as precursor of $\mathrm{P}$-chiral products.

\subsection{Amino Alcohols}

Amino alcohols have also been used as chiral auxiliaries for the synthesis of P-stereogenic compounds. In the early 1990s, Jugé and Stephan described the sequential ring-opening/methanolysis/nucleophilic substitution of (-)-ephedrine-derived oxazaphospholidine 3 using two distinct organolithium reagents. ${ }^{[17]}$

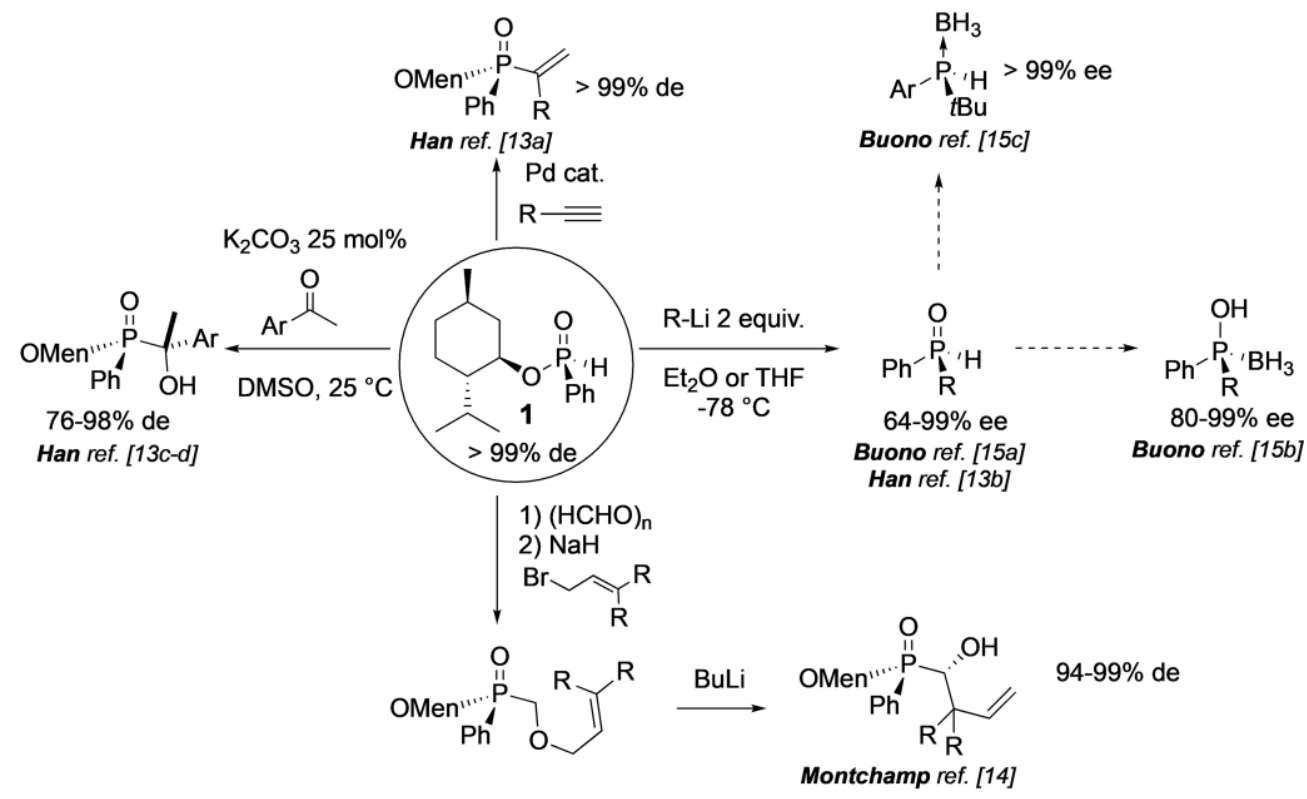



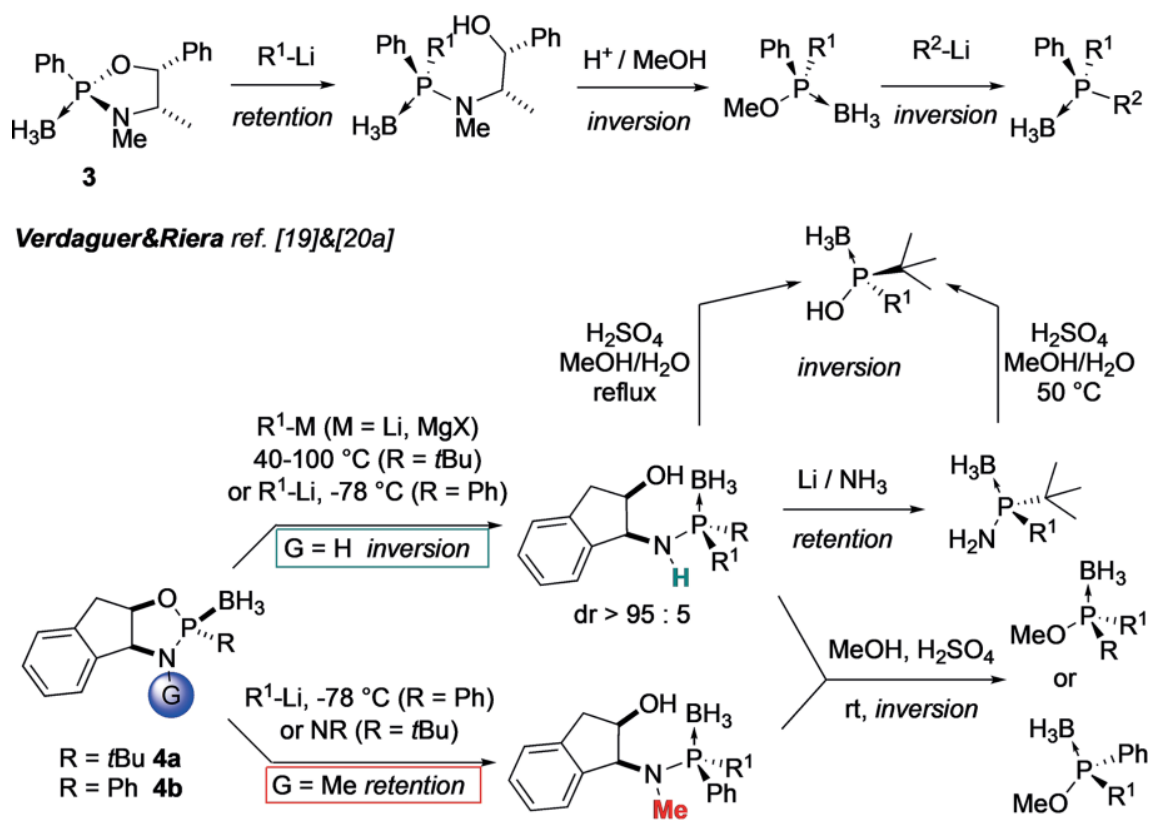

Scheme 4. Synthetic applications of oxazaphospholidine boranes.

A variety of alkyl and/or aryllithium reagent was found to be reactive, introducing diversity in the substitution pattern on the phosphorus atom (Scheme 4) ${ }^{[18]}$ However, this method cannot be applied to oxazaphospholidine containing bulky alkyl groups, due to the lack of reactivity of the precursor (of type $\mathbf{3}$ ) towards organolithium-mediated ring-opening. More recently, the group of Verdaguer and Riera has expanded this method to oxazaphospholidine containing more bulky substituents $(R=$ $t \mathrm{Bu}, \mathbf{4 a}$ ) on the phosphorus center, by using an aminoindanol as the chiral auxiliary. ${ }^{[19]}$

The reaction of the $\mathrm{NH}$ oxazaphospholidine with a variety of organometallics $\left(\mathrm{R}_{1}=\right.$ alkyl, aryl, or alkynyl) at $40-100{ }^{\circ} \mathrm{C}$ allowed the highly diastereoselective $(\mathrm{dr}>95: 5)$ formation of a diversity of (2-hydroxy)indanyl-1-aminophosphine-boranes with inversion of configuration at the phosphorus atom. ${ }^{[19 a]}$ These compounds could be reduced to the aminophosphine-boranes under Birch conditions with retention of configuration at the phosphorus atom, or could be hydrolyzed under acidic conditions with inversion of configuration. ${ }^{[20 a]}$ Interestingly, the reaction of oxazaphospholidine $\mathbf{4} \mathbf{b}$ led to an enantiodivergent nucleophilic substitution of organolithium reagents, depending on the nature of the $G$ substituent on the nitrogen. As reported with $\mathbf{4 a}$, inversion of configuration was observed with $\mathrm{G}=\mathrm{H}$, while retention of configuration occurred with $G=M e .{ }^{[19 b]} A c-$ cording to this procedure enantiopure tert-(butyl)methylphosphinous acid borane has been prepared, and the group of Verdaguer has shown that this compound can serve as a key precursor for the synthesis of P-stereogenic dialkylaminophosphine boranes. ${ }^{[20]}$ Also from this key intermediate, tert(butyl)methylphosphine borane has been synthesized, ${ }^{[21]}$ by using an analogous method to our group. ${ }^{[15 c]}$ In parallel to these works, oxazaphospholidines have also been employed for the synthesis of enantioenriched P-stereogenic phosphine oxides
(Scheme 5). Indeed, in 1993, the Brown group described the synthesis of various phosphine oxides, using ephedrine-substituted chloro oxazaphospholidine $\mathbf{5}$ (configurationally stable below $0{ }^{\circ} \mathrm{C}$ ) as the precursor. ${ }^{[22]}$ The $\mathrm{P}-\mathrm{Cl}$ bond was easily displaced in the presence of aryl Grignard reagents with retention of configuration. In situ oxidation of the corresponding P(III) compound with TBHP allowed the formation of the corresponding oxazaphospholidine oxide. These compounds could be transformed into P-stereogenic triarylphosphine oxide by using a similar strategy to Jugé and Stephan method. ${ }^{[17]}$ Later, our group described the ring-opening of prolinol-derived oxazaphospholidine 6 in the presence of tert-butyllithium via retention of configuration. Subsequent enantiodivergent acidolysis of the in situ generated aminophosphine allowed the synthesis of both enantiomers of tert-butyl(phenyl)phosphine oxide. ${ }^{[23]}$

More recently, the Han group has reported the use of an aminophenol-based auxiliary for the ring-opening of oxazaphosphine 2-oxide derivative $7 .{ }^{[24]}$ Unlike previous reports using oxazaphospholidines, the ring-opening of $\mathbf{7}$ occurred through $\mathrm{C}-\mathrm{N}$ bond cleavage before $\mathrm{C}-\mathrm{O}$ bond displacement. Thus, in the presence of a variety of organolithium reagents, the corresponding aryl phosphinates were formed in very high enantiospecificity with inversion of configuration at the phosphorus. The phenol leaving group could be easily displaced using either organolithium reagents ${ }^{[24 a]}$ or lithium amides, ${ }^{[24 b]}$ affording a wide array of enantiopure phosphine oxides and phosphinamides, respectively. A similar strategy has been used by Andrioletti and co-workers, using trans-1,2-aminocyclohex$a$ l $^{[25 a]}$ or D-Glucosamine $e^{[25 b]}$ as the chiral auxiliary.

\subsection{Chiral Amine}

Recently, Lopez Ortiz described the use of a chiral amine auxiliary for the diastereoselective ortho-lithiation/electrophilic trap- 


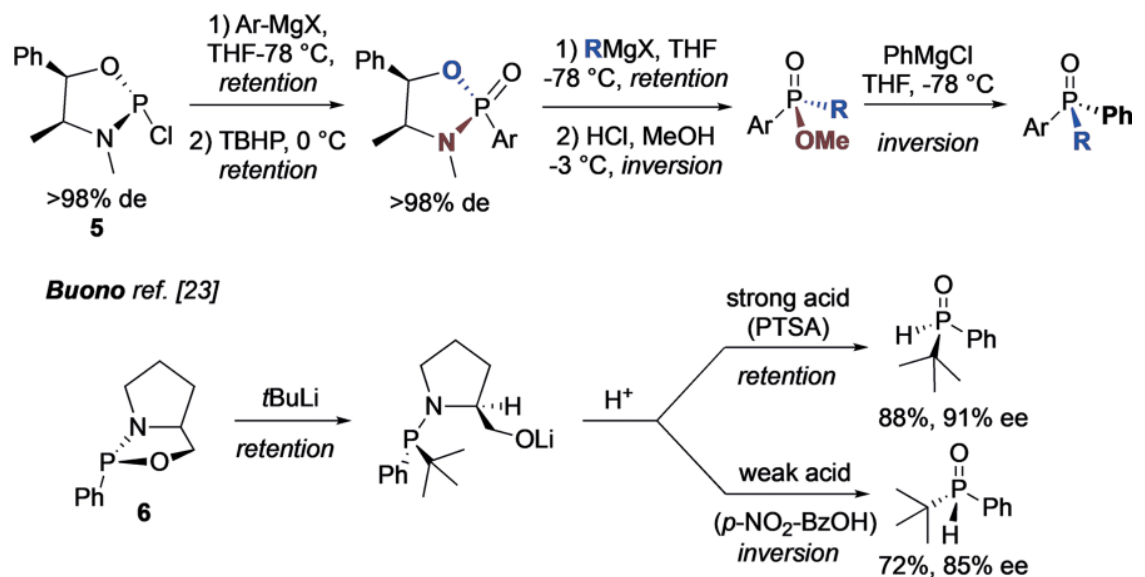

Z.S. Han ref. [24]

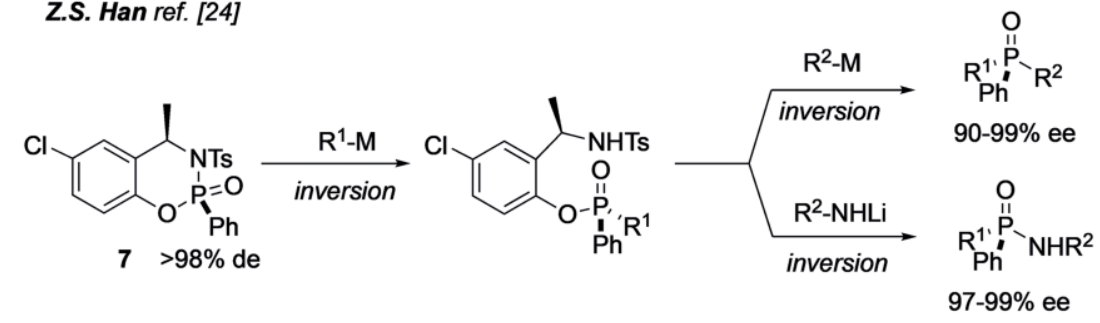

Andrioletti ref. [25a]

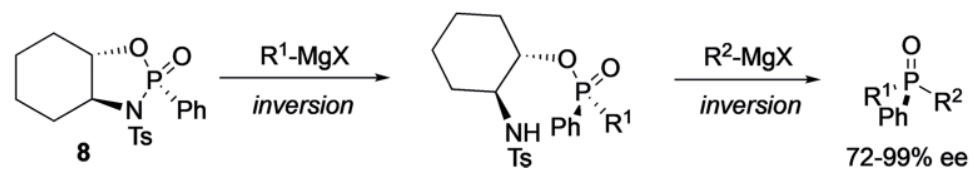

Scheme 5. Ring-opening of oxazaphospholidines for the synthesis of P-stereogenic phosphine oxides.

ping of diphenylphosphinamide 9 (Scheme 6). Using a wide array of halogen, oxygen, nitrogen, phosphorus, silicon, tin, and carbon-based electrophiles, the synthesis of P-stereogenic (aryl)(phenyl) phosphinamides was realized with very high diastereomeric ratios. ${ }^{[26]}$

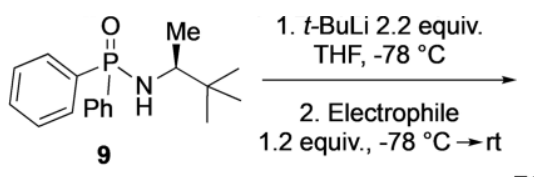

$\mathrm{FG}=\mathrm{Hal},[\mathrm{Si}],[\mathrm{Sn}], \mathrm{OH}, \mathrm{N}_{3}$

[C] or $\mathrm{P}(\mathrm{O}) \mathrm{Ph}_{2}$ with $\mathrm{dr}>91: 9$

Scheme 6. Diastereoselective ortho-lithiation of diphenylphosphinamides bearing a chiral amine.

\section{Synthesis of P-Stereogenic Compounds via a Chiral Stoichiometric Reagent}

\section{1 (-)-Menthol}

Like chiral auxiliaries, the use of a chiral stoichiometric reagent has proven to be a viable approach for access to enantioenriched P-stereogenic molecules. In particular, (-)-menthol was found to be a versatile platform for the synthesis of P-stereogenic compounds.
Indeed, the Gilheany group described the (-)-menthol-mediated dynamic kinetic resolution of in situ generated chlorophosphonium chlorides (Scheme 7) ${ }^{[27]}$ from either racemic phosphines ${ }^{[27 a]}$ or phosphine oxides. ${ }^{[27 b, c, d]}$ The key point of the success of this method was the rapid racemization of the chlorophosphonium chloride intermediate (especially when at least one aryl group is bound to phosphorus, as well as with small alkyl groups $\left.{ }^{[28]}\right)$, followed by the diastereoselective nu-

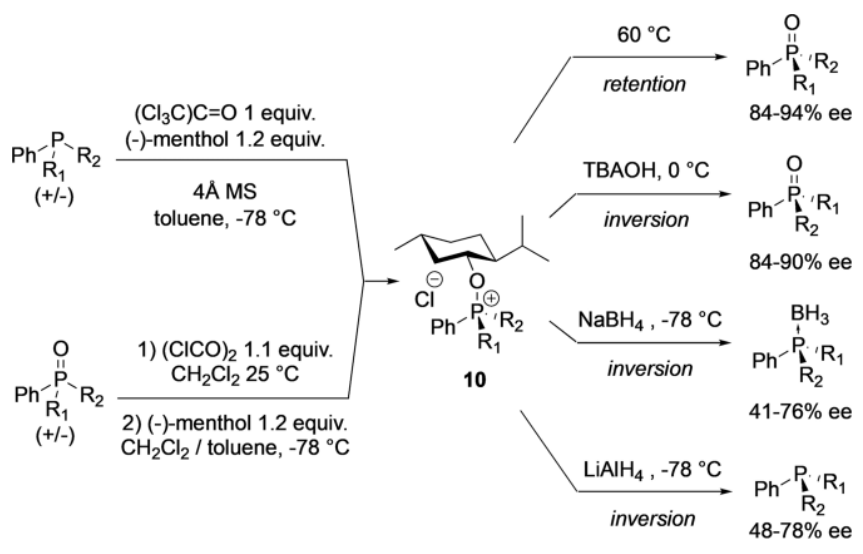

Scheme 7. Dynamic kinetic resolution of chlorophosphonium chlorides using (-)-menthol. 
cleophilic substitution of (-)-menthol to afford (-)-menthyloxy phosphonium chloride $\mathbf{1 0}$ which could be used a polyvalent precursor for the enantioconvergent synthesis of P-stereogenic $\mathrm{P}(\mathrm{III})$ and $\mathrm{P}(\mathrm{V})$ compounds. Indeed, when $\mathbf{1 0}$ was heated at $60{ }^{\circ} \mathrm{C}$ in acetonitrile Arbuzov-like process afforded the desired phosphine oxides with retention of configuration. ${ }^{[27 c]}$ The synthesis of P-stereogenic phosphine oxides was also amenable to an enantiodivergent process as $\mathbf{1 0}$ could react in the presence of tetrabutylammonium hydroxide to furnish the same products via inversion of configuration. ${ }^{[27 c]}$ Intermediate $\mathbf{1 0}$ also allowed the synthesis of enantioenriched P-stereogenic phosphines and phosphine-boranes through inversion of configuration at phosphorus, by using aluminum or boron hydride-based reductants, ${ }^{[27 b, 29]}$ respectively.

\section{$3.2(-)$-Sparteine and Surrogates}

The use of (-)-sparteine as a stoichiometric reagent for the enantioselective deprotonation of phosphorus compounds in the presence of organolithium reagents has seen many developments. In the 1990s, the groups of Evans ${ }^{[30]}$ and Imamoto ${ }^{[31]}$ reported the desymmetrization of (dimethyl) phosphineboranes through enantiotopic deprotonation using $n \mathrm{BuLi}$ or sBuLi as the bases (Scheme 8).

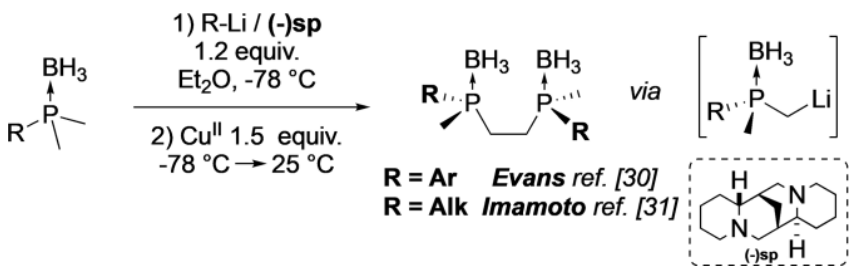

Scheme 8. (-)-sparteine-mediated enantioselective deprotonation of dimethylphosphine-boranes.

Since these early works, numerous developments of this approach have been made, allowing the access to a variety of enantioenriched phosphine-boranes ${ }^{[32]}$ and phosphine sulfides ${ }^{[33]}$ (Scheme 9). From compounds of type 11 and 12, enantioselec- tive deprotonation using (-)-sparteine yields the $S$ enantiomer of the deprotonated intermediate, which could be further functionalized with a range of electrophiles. ${ }^{[30,31,32 a-d, f, 33]}$ The access to the $R$ enantiomer of the deprotonated intermediate was enabled by the use of O'Brien (+)-sparteine surrogate. ${ }^{[32 e, g]}$ Enantioselective deprotonation has also been applied to $\mathrm{C}\left(\mathrm{sp}^{2}\right)-\mathrm{H}$ bonds, using a combination of (-)-sparteine and $n \mathrm{BuLi}^{[34]}$ (Scheme 10). In this method, the desymmetrization of diphenyl phosphinamides was realized in moderate enantioselectivities through enantiotopic ortho-lithiation/electrophilic trapping. (-)-Sparteine was also used in an elegant dynamic thermodynamic resolution of secondary phosphine-borane (SPB) 13, as described by the Livinghouse group ${ }^{[35]}$ (Scheme 11). Indeed, by using nBuLi as the base in combination with (-)-sparteine, the configurational instability of lithium tert-butyl(phenyl)phosphido-borane (Li-SPB) at $25^{\circ} \mathrm{C}$ could be exploited to develop an enantioconvergent synthesis of tertiary phosphine-boranes. Precipitation of a single diastereomer of the (-)-sparteine/Li-SPB complex allowed the thermodynamically driven dynamic resolution of tert-butyl-

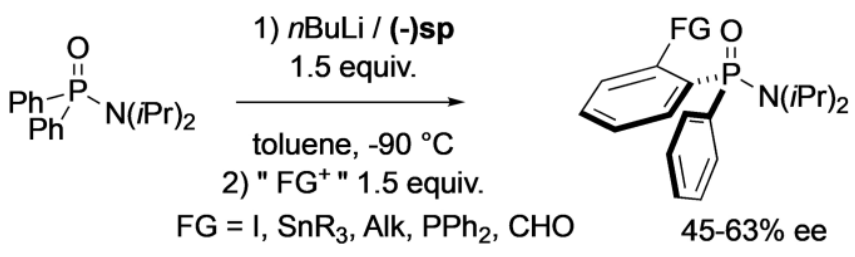

Scheme 10. Enantioselective ortho-lithiation of diphenylphosphinamides.

\section{Livinghouse ref. [35]}

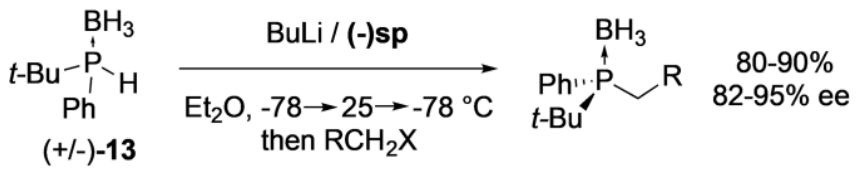

Scheme 11. (-)-sparteine mediated dynamic thermodynamic resolution of SPBs.

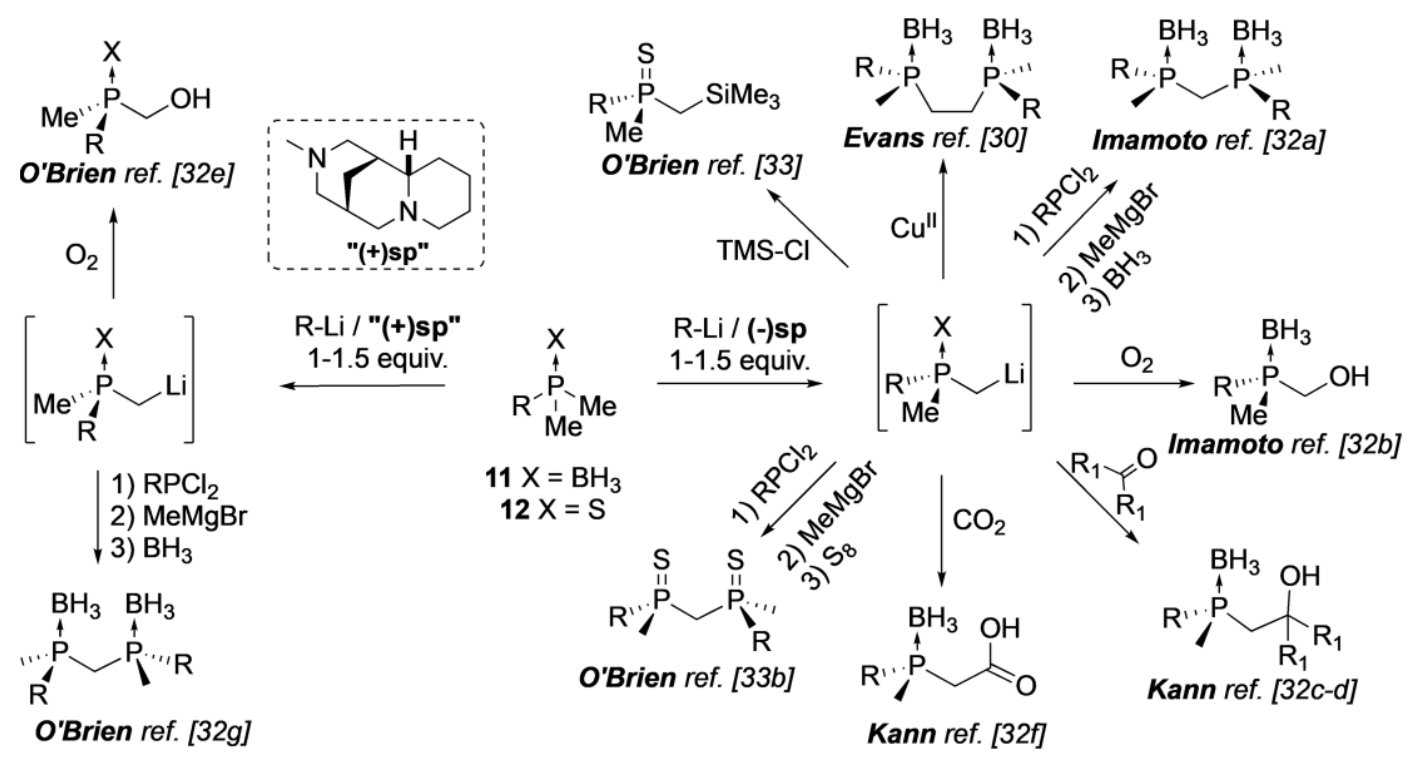

Scheme 9. Synthesis of P-chiral compounds via (-)-sparteine-mediated desymmetrization of C(sp $\left.{ }^{3}\right)-\mathrm{H}$ bonds. 
(phenyl)phosphine-borane with excellent enantiomeric excesses using a range of alkyl halide electrophiles.

\subsection{Dibenzoyltartaric Acid (DBTA)}

The dynamic thermodynamic resolution of tert-butyl(phenyl)phosphine oxide (SPO) has been realized under radical conditions in the presence of a stoichiometric amount of (-)-dibenzoyl tartaric acid (DBTA) with excellent enantiomeric excess (Scheme 12).

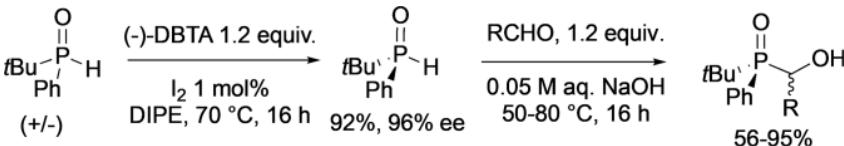

$$
\begin{aligned}
& \text { d.r. }>4.5: 1
\end{aligned}
$$

Scheme 12. DBTA-mediated highly stereoselective synthesis of $\alpha$-hydroxyalkylphosphine oxides.

Subsequent highly diastereoselective addition of the enantiopure SPO to aldehydes under thermodynamic control allowed the development of a highly enantio- and diastereoselective synthesis of $\alpha$-hydroxyalkylphosphine oxides, through a synthetic sequence consisting of two consecutive crystallization induced asymmetric transformations (CIAT) ${ }^{[36]}$

\section{Synthesis of P-Stereogenic Compounds via Catalytic Enantioselective Methods ${ }^{[37]}$}

In the last two decades, the development of catalytic and enantioselective methods for the synthesis of P-stereogenic compounds has emerged as a powerful strategy for the preparation of such chemicals. Although most of the work has focused on the use of chiral transition metal catalysts, a few examples of organocatalyzed processes have recently been reported.

\subsection{Transition-Metal Catalyzed Dynamic Kinetic Resolution of Secondary Phosphines and SPOs}

Historically, the use of phosphorus-borane compounds for the preparation of optically pure P-stereogenic molecules through enantio- or diastereospecific metal-catalyzed couplings has been described ${ }^{[38,39]}$ (Scheme 13). Indeed, Imamoto ${ }^{[38]}$ and Livinghouse ${ }^{[39]}$ reported the stereospecific palladium-catalyzed P-arylation of secondary phosphinite-boranes and secondary phosphine-boranes, respectively. Interestingly, in the case of enantio- and diastereo-pure $O$-menthyl secondary phosphiniteborane, a solvent-mediated stereodivergence was observed. While THF provided the desired product with retention of configuration at the phosphorus atom, more polar and coordinating solvents such as acetonitrile yielded the other diastereomer of the product, suggesting a complete epimerization of the deprotonated species. Livinghouse and co-workers described the enantiospecific palladium-catalyzed P-arylation of methyl(phenyl)phosphine- borane, from the corresponding in situ generated copper phosphide-borane, via retention of configuration at the phosphorus center.

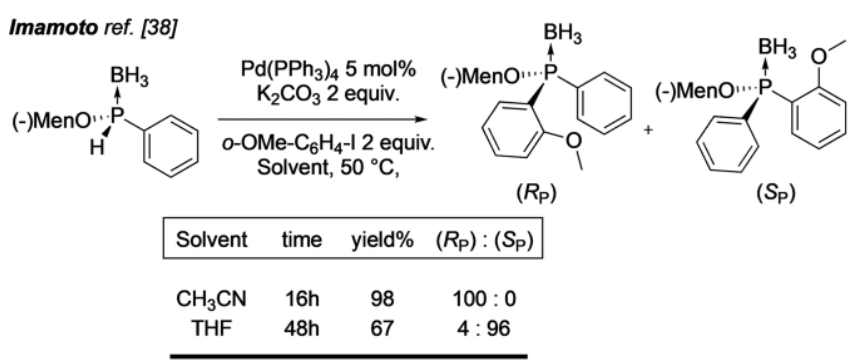

Livinghouse ref. [39]

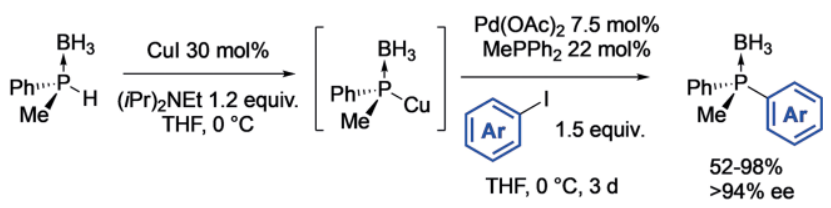

Scheme 13. Stereospecific P-arylations of enantiopure phosphorus-borane compounds.

An interesting insight into the reaction mechanism in the enantiospecific P-arylation was brought by Glueck (Scheme 14). ${ }^{[40]}$ Indeed, the authors synthesized $(S, S)$-chiraphos $\mathrm{Pd}(o-\mathrm{An})(I)$ complexes, and the stoichiometric reaction of this complex in the presence of the $(R)$ and $(S)$ enantiomers of sodium methyl(phenyl)phosphide borane was performed. While the transmetallation was found to be enantiospecific (occurring with retention of configuration at the phosphorus atom) at $-78{ }^{\circ} \mathrm{C}$ (entries $1-2$ ), substantial epimerization of the phosphorus atom was observed at room temperature (entries 3-4). Like the transmetallation step, the reductive elimination was also found to occur stereospecifically with retention of configuration (entries 6-9). However, these compounds were not found to be successful precursors for the enantioselective P-arylation through dynamic kinetic resolution DKR, according to Glueck's work (entry 5) as well as other studies. ${ }^{[41]}$ Nevertheless, the dynamic kinetic resolution of racemic secondary phosphines has been realized using a range of transition-metal-catalyzed reactions. Indeed, by taking advantage of the rapid racemization of transition metal phosphides (especially the ones bearing at least one aromatic ring on the phosphorus atom), ${ }^{[42]}$ highly enantioselective methods have been developed (Scheme 15). Firstly, the enantioselective P-arylation of secondary phosphines has been realized by Glueck ${ }^{[43]}$ by means of palladium catalysis, using bulky aryl-substituted methyl (aryl)phosphines. A similar strategy was developed by Toste ${ }^{[44]}$ using silicon-substituted phosphines as secondary phosphine surrogates. Likewise, the P-alkylation was performed using chiral platinum ${ }^{[45]}$ or ruthenium ${ }^{[46]}$ catalysts. The dynamic kinetic resolution of secondary phosphines has also been realized through platinum-catalyzed conjugate addition, albeit with lower enantioselectivity. ${ }^{[4]}$ More recently, Hayashi reported an original palladium(II)-catalyzed enantioselective oxidation of secondary diarylphosphines through conjugate addition to quinone derivative. ${ }^{[48]}$ This allowed the preparation of a range of P-stereogenic phosphinite compounds with moderate to very high enantioselectivities (24-98\% ee).

The enantioselective P-arylation of racemic (alkyl)(aryl) secondary phosphine oxides has been realized by Gaunt in 2016 ${ }^{[49]}$ 


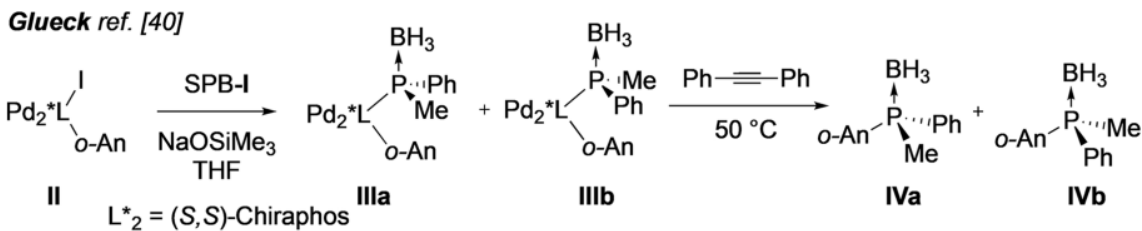

\begin{tabular}{|cccc|}
\multicolumn{4}{c}{ Transmetallation } \\
\hline entry & $\mathrm{T}\left({ }^{\circ} \mathrm{C}\right)$ & ee $(\mathrm{I})$ & Illa:IIIb \\
\hline 1 & -78 & $95(R)$ & $97: 3$ \\
2 & -78 & $99(S)$ & $3: 97$ \\
3 & 21 & $95(R)$ & $93: 7$ \\
4 & 21 & $97(S)$ & $18.5: 81.5$ \\
5 & 21 & $r a c$ & $61.5: 18.5$ \\
\hline
\end{tabular}

Reductive Elimination

\begin{tabular}{cccc|}
\hline entry & $t(h)$ & IIla:IIIb & IVa:IVb \\
\hline 6 & 72 & $97: 3$ & $95.5: 4.5$ \\
7 & 96 & $97: 3$ & $93.5: 6.5$ \\
8 & 37 & $0: 100$ & $1: 99$ \\
9 & 48 & $7: 93$ & $3.5: 96.5$ \\
\hline
\end{tabular}

Scheme 14. Mechanistic insights on the stereo-specific P-arylation of secondary phosphine-boranes.

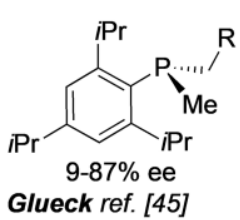
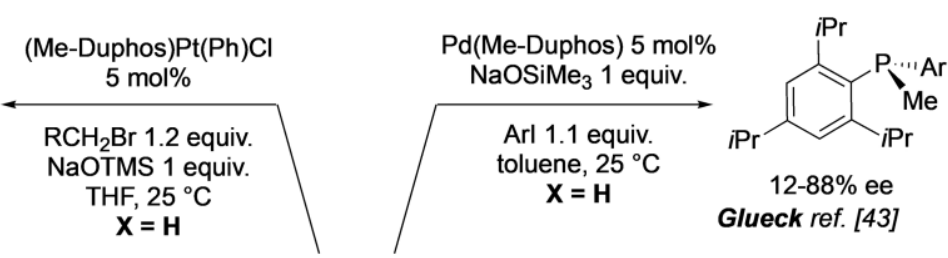<smiles>CCCCN(C)c1c(C(C)C)cc(C(C)C)cc1C(C)C</smiles>

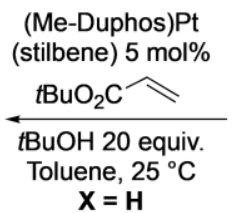

$58 \%$ ee

Glueck ref. [47]

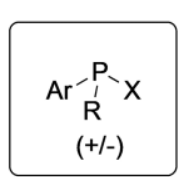

$(+/-)$

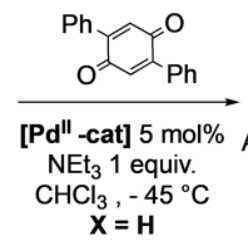

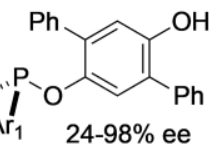

Hayashi ref. [48]

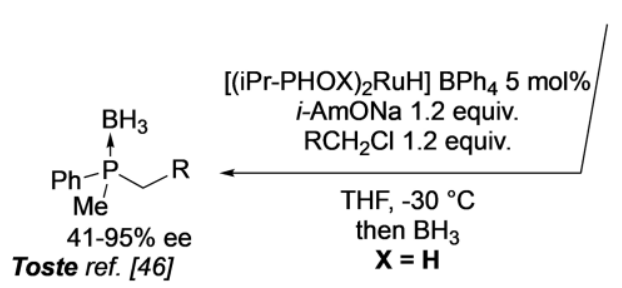

Toste ref. [46]

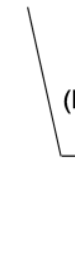

(FerroTANE)PdCl 5 mol\% $\mathrm{Ar}-\mathrm{Br}$

DMPU, $60^{\circ} \mathrm{C}$ then $\mathrm{BH}_{3}$ or $\mathrm{S}_{8}$ $\mathbf{X}=$ TIPS

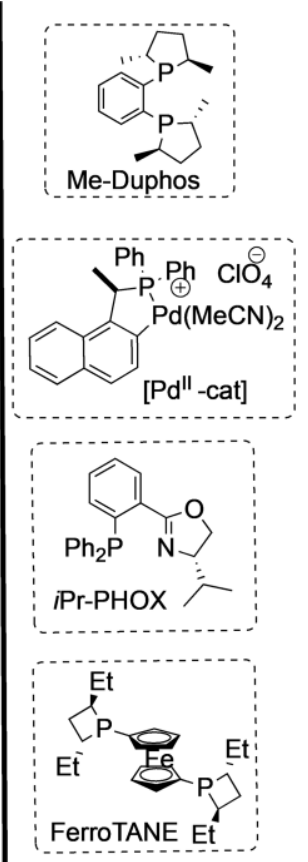

Scheme 15. Transition-metal catalyzed DKR of secondary phosphines.

by using a copper(II)-PyBox catalytic system and diaryliodonium salts as the aryl electrophiles. The corresponding diaryl(alkyl)phosphine oxides could be obtained in excellent yields and enantioselectivities (Scheme 16a). Interestingly, the starting SPOs recovered after the reaction was found to be racemic. However, the reaction of the enantiopure SPO was found to occur with different selectivity following the "matched" or "mismatched" chirality of the catalyst, which suggests that the reaction might be more complex than a simple DKR (Scheme 16a).

In addition, the racemization of complexed phosphinito is usually difficult, as these species are known to be more configurationally stable than those of the corresponding complexed phosphido. ${ }^{[42 \mathrm{~b}]}$ However, it has been recently shown that they can readily racemize under radical conditions. ${ }^{[36]}$ Therefore, a potential radical intermediate involved in the formation of the copper (III) species might account for the observed partial race- mization of the SPO starting material. A similar approach was developed by Cai using a chiral palladium catalyst in the presence of 0 -aminoaryl iodides as the electrophilic partners (Scheme 16b). ${ }^{[50]}$ When using diaryl phosphine oxides, moderate to good enantioselectivities were obtained with P-Phos as the chiral ligand, while phenyl (methyl)phosphine oxide yielded the corresponding products in low enantioselectivity (19\% ee) in the same conditions. A more general version of this reaction was realized in late 2019 by Zhang using aryl(alkyl) SPOs using XiaoPhos, a sulfinamide-substituted monophosphine, as the chiral ligand (Scheme 16c). ${ }^{[51]}$ Using this approach, a wide array of alkyl(aryl)phosphine could be efficiently transformed. Quite remarkably, the nature of the alkyl group bound to the phosphorus did not affect the selectivity of the reaction, even though bulky tert-butyl and neo-pentyl substituents gave the lowest enantioselectivities ( $81 \%$ ee and $88 \%$ ee, respectively). 

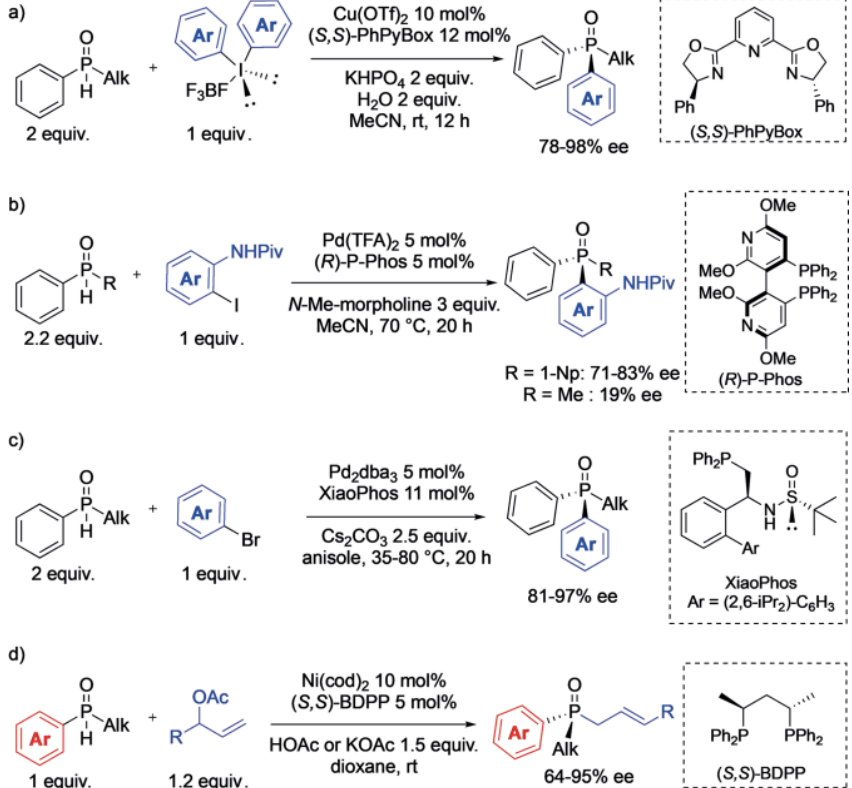

Scheme 16. Enantioselective transformation of racemic SPOs.

On the other hand, a broad scope of ortho-, meta- and parasubstituted aryl bromides could be introduced without any detrimental effect on the enantioselectivity. Besides, Zhang and co-workers designed a nickel-catalyzed enantioselective allylic substitution of SPOs nucleophiles with a wide array of substituted allylic acetates, using (S,S)-BDPP as the chiral ligand (Scheme 16d). ${ }^{[52]}$ Interestingly, the nature of the additive had a huge effect on the stereochemical outcome of the reaction. While the use of a strong inorganic base such as potassium phosphate resulted in clean kinetic resolution, weaker bases such as potassium acetate or acetic acid enabled the DYKAT of the secondary phosphine oxide (with alk $=\mathrm{Me}$ and $\mathrm{R}=\mathrm{Ph}$, the product was obtained in $96 \%$ yield and $87 \%$ ee).

\subsection{Transition-Metal Catalyzed Desymmetrization of Vinyl and Alkynyl Phosphine Oxides}

Another strategy for the synthesis of P-stereogenic compounds through enantioselective transition-metal catalysis relies on the desymmetrization of achiral alkynyl (Scheme 17) or vinyl (Scheme 18) substituted phosphorus oxides. Indeed, in 2008, Tanaka and co-workers developed a rhodium-catalyzed enantioselective desymmetrization, a [2+2+2] cycloaddition of bis(alkynyl) phosphine oxides. ${ }^{[53 a]}$ While this reaction was found to be very selective when using small alkyl group $\left(R_{1}=M e, 87-\right.$ $95 \%$ ee), the use of more hindered substrates resulted in largely decreased enantioselectivities $\left(50 \%\right.$ ee with $\mathrm{R}_{1}=\mathrm{Ph}$, $41 \%$ ee with $R_{1}=t B u$ ). Very recently, Zhou et al. have reported a highly enantioselective synthesis of diverse P-chiral tertiary phosphines oxides bearing an acetylene group via a $\mathrm{Cu}(\mathrm{I})$ catalyzed Huisgen [3+2] cycloaddition. In this work, chiral pyridinebisoxazolines (PyBOx) ligands bearing a bulky C4 shielding group played an important role in achieving excellent enantioselectivity while suppressing side bis-triazoles. Using this ligand, the desymmetrization of prochiral di(ethynyl)phosphine oxides
(75-96\% ee) and di(ethynyl)phospholes (92-99\% ee) was realized. ${ }^{[53 \mathrm{~b}]}$ Interestingly, this ligand was also able to perform the enantioselective Huisgen [3+2] cycloaddition on racemic mono(alkynyl)phosphine oxides via kinetic resolution with good to excellent selectivity $(12 \leq s \leq 116) .{ }^{[53 b]}$ More recently, a gold-catalyzed cycloisomerization of bis(ortho-hydroxyphenyl) (alkynyl)phosphine oxides and bis(alkynyl)(ortho-hydroxyphenyl) phosphine oxides was reported by $\mathrm{Zi}^{\left[{ }^{[54]}\right.}$

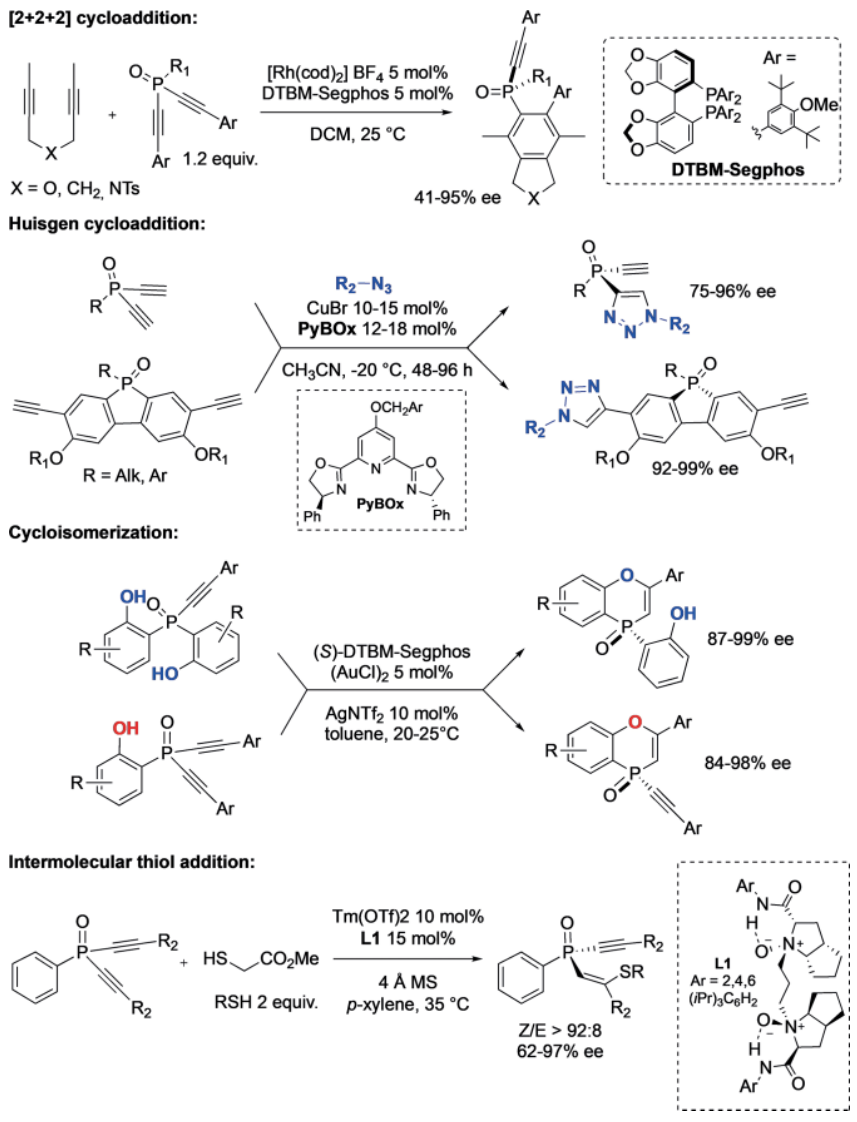

Scheme 17. Desymmetrization of bis(alkynyl)phosphorus compounds through $\pi$-activation.

Ring-closing metathesis (RCM):
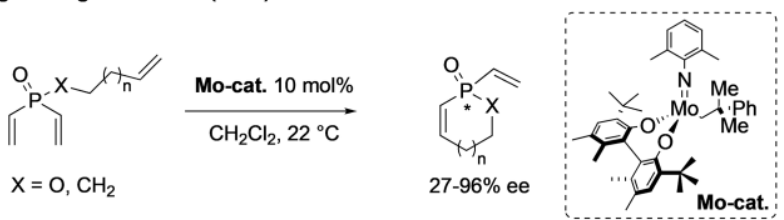

"Transfer" hydroarylation:

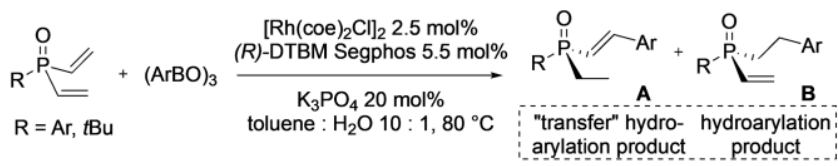

$$
\begin{aligned}
& \text { up to } \mathbf{A}: \mathbf{B}>99: 1 \\
& 60-95 \% \text { ee }
\end{aligned}
$$

Scheme 18. Desymmetrization of divinyl phosphorus oxides.

A similar thulium (III)-catalyzed intermolecular process was reported by Liu and Feng very recently. In their work, the authors performed a diastereo- and enantioselective Z-selective 
addition of methyl 2-mercaptoacetate to (dialkynyl)phosphine oxides using chiral N, $\mathrm{N}^{\prime}$ dioxide ligand L1. ${ }^{[55]}$ When an alkyl group was present on the phosphorus atom instead of $\mathrm{Ph}$ group, the enantioselectivity decreased slightly $(86 \%$ ee with $\mathrm{R}=t \mathrm{Bu}, 48 \%$ ee with $\mathrm{R}=\mathrm{Me}$, compared with $97 \%$ ee using the same substrate having $\mathrm{R}=\mathrm{Ph}$ ). The enantioselective desymmetrization of divinyl phosphorus oxide derivatives has also been reported using transition-metal catalysis (Scheme 18).

In 2009, the desymmetrization of olefin-tethered divinylphosphinates and divinylphosphine oxides was realized by Hoveyda and Gouverneur through an enantioselective ring-closing metathesis (RCM) using a chiral molybdenum catalyst. ${ }^{[56]}$ Recently, Hayashi reported an unusual rhodium-catalyzed enantioselective "transfer" hydroarylation of divinylphosphine oxides. ${ }^{[57]}$ In this work, highly enantioselective reactions were achieved using (R)-DTBM Segphos as the ligand. Interestingly, the "transfer" hydroarylation product (consisting of a domino Heck-type reaction/Rh-H addition to the least hindered electron-poor olefin) was largely favored compared to the classical hydroarylation product. Very recently, Vidal-Ferran and co-workers have also described the rhodium-catalyzed hydrogenative kinetic resolution of vinylic phosphine oxides and phosphinates (Scheme 19). ${ }^{[58]}$ Both the desired alkyl phosphorus oxides ( $25 \leq \mathrm{s} \leq 217$ ), as well as the starting vinylic phosphorus oxides ( $22 \leq \mathrm{s} \leq 213$ ), could be obtained with a high level of selectivity.

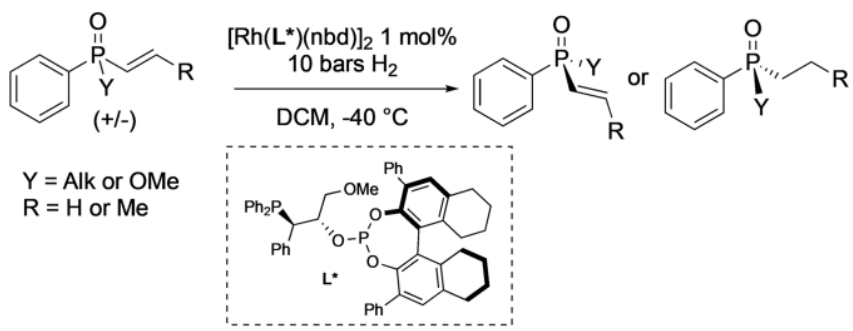

Scheme 19. Kinetic resolution of tertiary vinyl phosphine oxides through enantioselective hydrogenation.

\subsection{Transition-Metal Catalyzed C-H Functionalizations}

The enantioselective synthesis of P-stereogenic molecules has also been realized through metal-catalyzed $\mathrm{C}\left(\mathrm{sp}^{2}\right)-\mathrm{H}$ bond functionalizations. ${ }^{[59]}$ This approach was used to perform a wide array of desymmetrization reactions of various achiral diarylphosphorus oxides, such as diarylphosphinamides, ${ }^{[59 a, c-e]}$ diarylphosphonates ${ }^{[59 b]}$ and diarylphosphine oxides. ${ }^{[59 f, g]}$

Using palladium (0) catalysis (Scheme 20), the intramolecular $\mathrm{C}-\mathrm{H}$ arylation of $\mathrm{N}$-(o-bromophenyl)- $\mathrm{N}$-alkyl diphenyl phosphinamide was realized in high enantioselectivities by Duan and coworkers, using TADDOL-phosphoramidite $\mathbf{L} 2$ as the chiral ligand. ${ }^{[59 a]}$ A similar approach was used by the group of Tang to perform an enantioselective intramolecular $\mathrm{C}-\mathrm{H}$ arylation of diaryl (o-bromoaryl) phosphonates using (R)-Antphos (L3) as the ligand. ${ }^{[59 b]}$ Interestingly, the corresponding enantioenriched cyclic P-stereogenic phosphonates could be reacted sequentially in the presence of two distinct organolithium reagents to provide biarylphosphine oxides in a stereodivergent fashion, following the order of addition of both organolithium reagents.
The direct intermolecular $\mathrm{C}-\mathrm{H}$ arylation of phosphinamides has been realized by means of palladium(II) catalysis, in the presence of aryl(pinacol)boronic esters, using $\mathrm{N}$-protected amino acid L4. This method allowed the highly enantioselective preparation of biarylphosphinamides. ${ }^{[59 c]}$ The use of noble group 9 metal catalysts such as rhodium (III) and iridium (III) bearing chiral C $\mathrm{Cp}^{*}$-type chiral ligands was developed later on by the Cramer group. ${ }^{[59 \mathrm{~d}-\mathrm{g}]}$ Indeed, the Rh-catalyzed enantioselective ortho- $\mathrm{C}\left(\mathrm{sp}^{2}\right)-\mathrm{H}$ alkenylation/cyclization of achiral or racemic phosphinamides allowed the preparation of highly enantioenriched P-stereogenic cyclic phosphinamides via desymmetrization $^{[59 d]}$ (86-92 \% ee) or kinetic resolution ${ }^{[59 e]}$ ( $s \leq 49$ ), respectively (Scheme 21). The iridium-catalyzed enantioselective synthesis of phosphine oxides has also been realized by the same group. These methods rely on the ability of $\mathrm{Cp}^{*}$-iridium (III) nitrenes and carbenes to perform $\mathrm{C}-\mathrm{H}$ insertion into $\mathrm{C}\left(\mathrm{sp}^{2}\right)-\mathrm{H}$ bonds (Scheme 22).

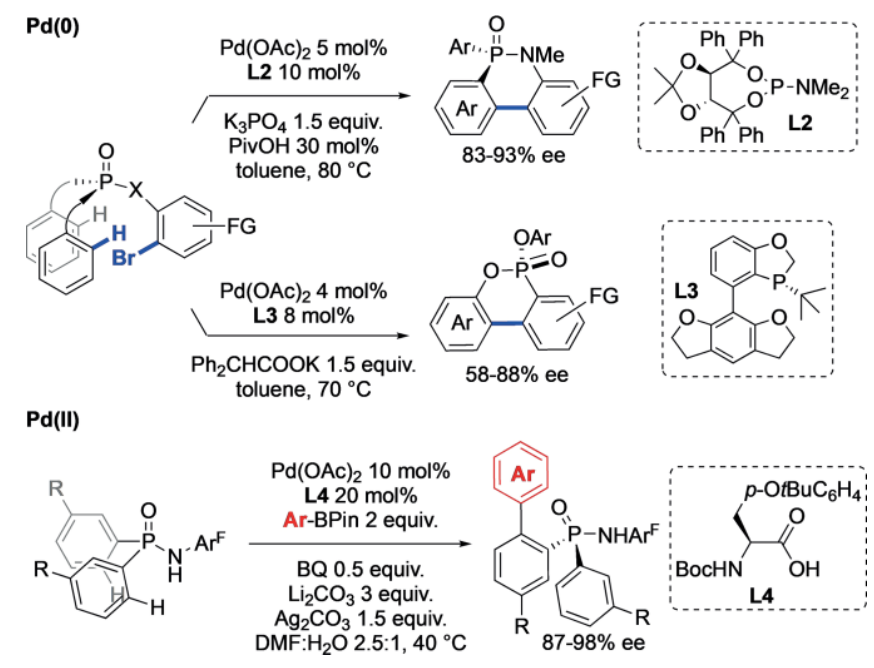

Scheme 20. Synthesis of P-stereogenic phosphorus oxides through Pd-catalyzed $\mathrm{C}-\mathrm{H}$ functionalizations.

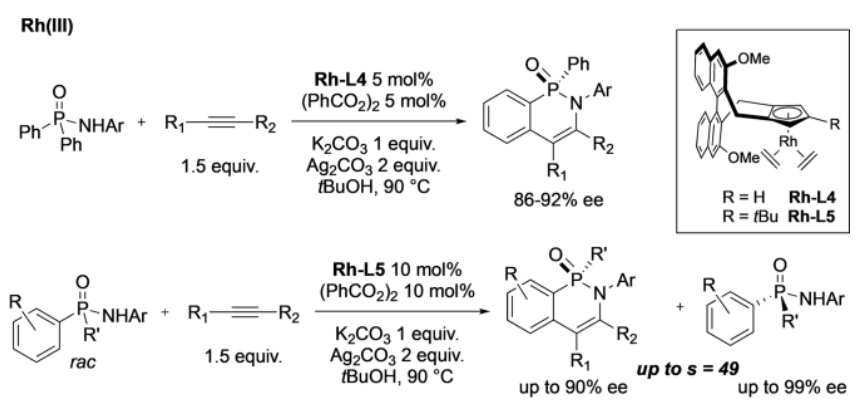

Scheme 21. Rh-catalyzed enantioselective ortho-C-H alkenylation/cyclization.

Particularly, a strong synergistic effect between the use of the chiral C p $^{*}$-type ligand L4 and a chiral carboxylic acid A1 was observed. According to this method, the highly enantioselective ortho-amination and ortho-arylation of phosphine were realized, using azides ${ }^{[59 f]}$ and diazo compounds $s^{[59 g]}$ as the nitrene and carbene sources, respectively. 


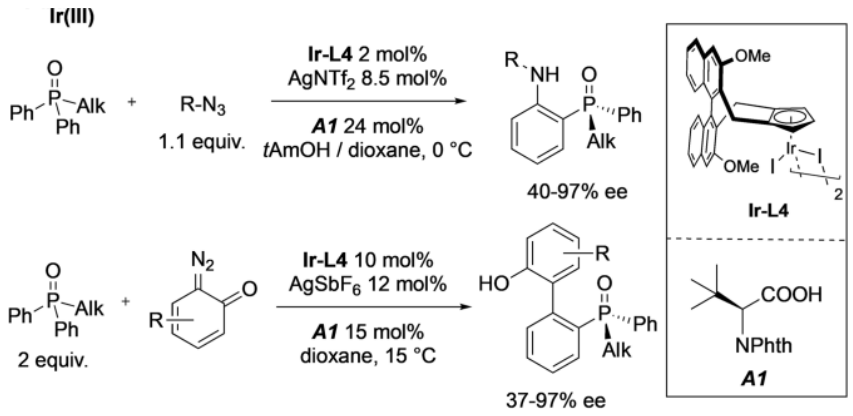

Scheme 22. Ir-catalyzed enantioselective synthesis of P-chiral phosphine oxides via ortho-C-H functionalization.

\subsection{Organocatalytic Processes}

Unlike transition-metal catalyzed methods, the use of organocatalysts in the context of the enantioselective synthesis of P-stereogenic molecules has seen much fewer developments. Early work from the Lebel group demonstrated that enantioselective phase-transfer catalysis for the DKR of phenylphosphido-borane was possible, although using Cinchona alkaloidderived ammonium as the catalyst, the alkylation product (using Mel as electrophile) was afforded in very modest enantioselectivity (17\% ee). ${ }^{[60]}$ Among the rare examples of highly enantioselective organocatalyzed processes, recent works on the desymmetrization of di(ortho-hydroxyphenyl) phosphorus oxides have been reported (Scheme 23). ${ }^{[61]}$ In 2016, Song and Chi reported a desymmetrizative NHC-catalyzed O-acylation of di(ortho-hydroxyphenyl) phosphinamides and di(ortho-hydroxyphenyl) phosphinates using a triazolium-based NHC catalyst. ${ }^{[61 a]}$ According to this method, a range of aromatic aldehydes could be used as acylation reagents (thanks to the in situ oxidation of the Breslow intermediate, which generates the electrophilic acyl triazolium species).

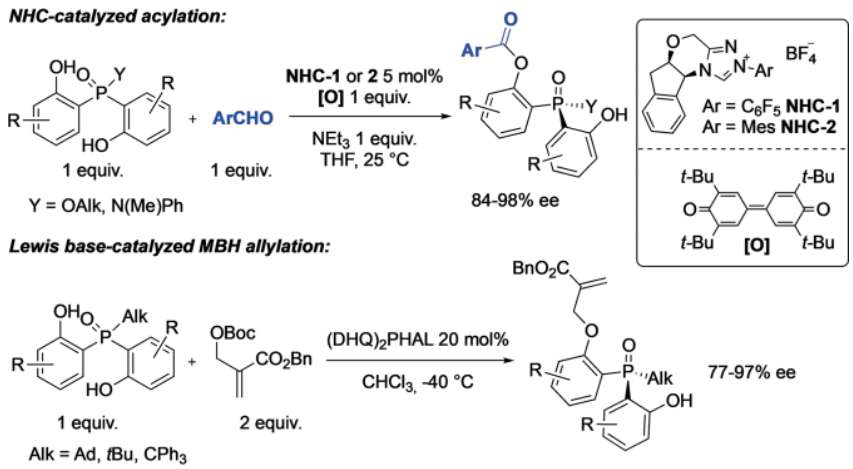

Scheme 23. Desymmetrization of di(ortho-hydroxyphenyl) phosphorus oxides via organocatalysis.

However, this reaction was not found to be very efficient for the preparation of enantioenriched phosphine oxides, as the enantioselectivity dropped substantially when using this type of starting material ( $42 \%$ ee when $\mathrm{Y}=\mathrm{Ph}$ ). More recently, the desymmetrization of analogous phosphine oxides has been realized by a Lewis base-catalyzed Morita-Baylis-Hillman (MBH) allylation using $(\mathrm{DHQ})_{2} \mathrm{PHAL}$ as the catalyst. ${ }^{[61 \mathrm{~b}]}$ In this report, a series of prochiral phosphine oxides featuring a bulky alkyl group $\left(\mathrm{Alk}=t \mathrm{Bu}, \mathrm{Ad}\right.$, and $\left.\mathrm{CPh}_{3}\right)$ could be converted with good to excellent enantiomeric excesses.

\section{Resolution of P-Stereogenic Compounds via SemiPreparative Chiral Separation}

Our group has recently developed the use of semi-preparative chiral HPLC for the isolation of enantiopure P-stereogenic precursors. Indeed, a range of adamantyl (aryl) and (alkyl) H-phosphinates could be separated on a multigram scale. ${ }^{[62]}$ Interestingly, the reaction of adamantyl (phenyl) $\mathrm{H}$-phosphinate in the presence of tert-butyllithium in THF resulted in higher enantioselectivity than that of its (-)-menthyl $\mathrm{H}$-phosphinate equivalent. This is due to the reduced nucleophilicity of the adamantyl oxide anion when compared with the menthyl oxide anion, which virtually suppresses the racemization of the phosphinate precursor in the presence of alkoxide anion generated in the medium (Scheme 24). ${ }^{[62 \mathrm{~b}]}$

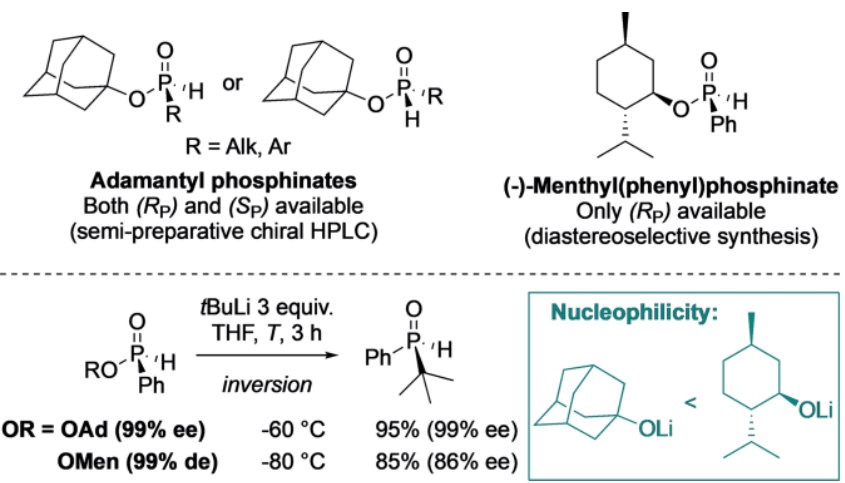

Scheme 24. O-(-)-Menthylphosphinate vs. O-adamantyl phosphinate in the stereoselective addition of R-Li.

Since our initial contribution, we have continued exploiting the synthetic potential of $\mathrm{H}$-adamantyl phosphinates for the synthesis of enantioenriched P-stereogenic molecules (Scheme 25). In the seminal report, we synthesized an array of (hydromethyl) adamantyl H-phosphinates through a basecatalyzed addition of the corresponding $\mathrm{H}$-phosphinates to formaldehyde. These compounds could be easily reduced with borane at room temperature through inversion of configuration at the phosphorus center to generate a range of enantiopure adamantyl (hydroxymethyl)phosphinite-boranes. The nucleophilic substitution of the $\mathrm{O}$-adamantyl leaving group in the presence of organolithium reagents was also studied in this article. An extension to this work was also carried out by our research group in order to perform the one-pot synthesis and reduction of $\alpha$ - and $\beta$-hydroxyalkyl phosphine oxides. ${ }^{[63 a]}$ Interestingly, the borane-mediated reduction of $\alpha$-hydroxyalkyl phosphine oxides was much faster than that of $\beta$-hydroxyalkyl phosphine oxides. This observation prompted us to postulate a cyclic intermediate in which the oxygen of the $\mathrm{P}=\mathrm{O}$ bond interacts with the boron atom after deprotonation of $\mathrm{OH}$ group with $\mathrm{BH}_{3}$.

Finally, the reactivity of the synthesized hydroxyalkyl phosphine-boranes was studied. Particularly, $\alpha$-hydroxyalkyl phos- 


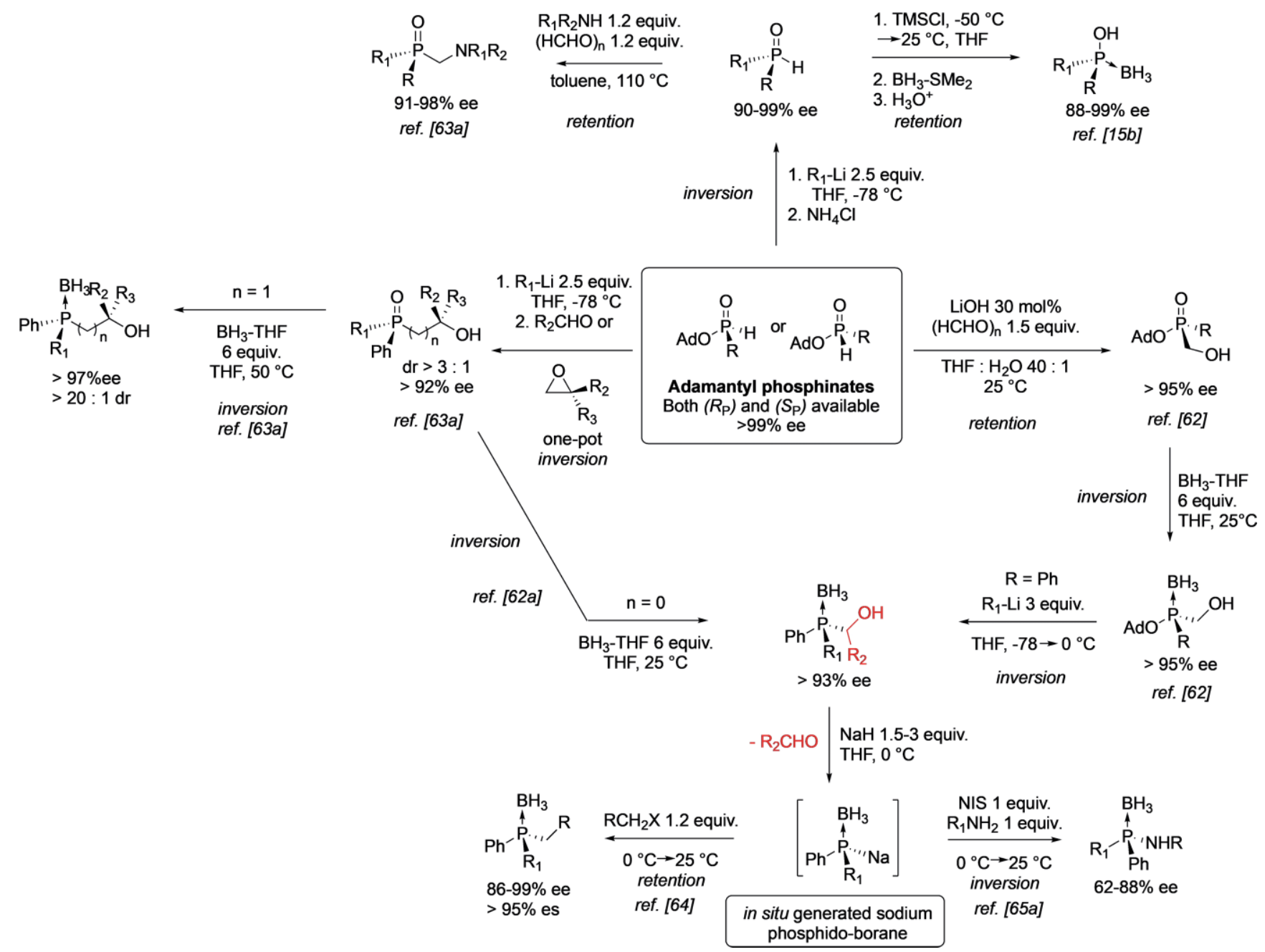

Scheme 25. $\mathrm{H}$-adamantylphosphinates as universal precursors to P-stereogenic compounds (obtained from the $\left(S_{\mathrm{P}}\right)$-phosphinate).

phine-boranes underwent decarbonylation under basic conditions, allowing the in situ generation of configurationally unstable alkali phosphide boranes. Slow generation of these phosphorus nucleophiles in the presence of an electrophile such as an alkyl halide ${ }^{[64]}$ or an electrophilic halide source such as $\mathrm{NIS}^{[65 a]}$ allowed the nearly room-temperature, highly enantiospecific formation of various phosphine-boranes ${ }^{[64]}$ and aminophosphine-boranes. ${ }^{[65 a]}$ This protecting group strategy thus allowed to circumvent the configurational instability of these anionic species, by taking advantage of the slow generation of the phosphide, together with its fast reaction in the presence of excess electrophile in the media.

\section{Resolution of P-Stereogenic Compounds via the Formation of Diastereomeric Complexes}

In this Chapter, we will only consider the resolution of neutral organophosphorus compounds having neither acid nor basic functional groups, which may be resolved in high optical purity by direct interaction of the phosphorus function with the re- solving agent. ${ }^{[66]}$ We have seen in Chapter 3.3 that the tertbutyl(phenyl)phosphine oxide (SPO) form complexes with the dibenzoyl tartaric acid (DBTA). It has also been applied to the resolution of diphosphine oxides using the same protocol (Scheme 26). ${ }^{[67]}$ After the synthesis of the $d l$-isomers, their complexation with (+)-DBTA allowed their precipitation. After washing with aqueous basic solution, extraction and concentration, the enantiopure diphosphine oxides were obtained in high optical purities (>98\%) and in moderate yields. The example of Liu and Zhang ${ }^{[67 d]}$ is very interesting as they have described the subsequent treatment of the remainder liquor with the other DBTA enantiomer to give the opposite diphosphine oxide enantiomer in $34 \%$ yield and $99 \%$ ee. For all of these examples, the subsequent stereospecific reductions of the $\mathrm{P}=\mathrm{O}$ functions ${ }^{[68]}$ led to enantiopure bis-P-stereogenic diphosphines. Imamoto ${ }^{[67 \mathrm{~b}, \mathrm{~d}]}$ and Zhang ${ }^{[67 \mathrm{e}]}$ showed that the latter are very efficient as asymmetric ligands in Rh-catalytic asymmetric hydrogenation (high enantioselectivities > $99 \%$; and reactivities up to 10000 TON). These selected examples illustrate the interest to realize the resolution of phosphine oxide easily reduced in phosphine rather a direct resolution of phosphines involving expensive transition metals. 
Hamada ref. [67a]

$\left(S_{p}, S_{p}\right) 39 \%, 99.4 \%$ ee

Schmutzler ref. [67c]<smiles>CC(C)(C)[PH](=O)(c1ccccc1)c1cccc2cccc(P(=O)(O)c3ccccc3)c12</smiles>

$\left(S_{P}, S_{P}\right) 30 \%,\left[\square_{D}^{20}=-184^{\circ}\left(0.3 \mathrm{~mol} / \mathrm{L}\right.\right.$ in $\left.\mathrm{CH}_{2} \mathrm{Cl}_{2}\right)$
Imamoto ref. [67b]<smiles>CC(C)PP(C)(=O)c1ccccc1P(C)(=O)C(C)C</smiles>

$\left(R_{\mathrm{P}}, R_{\mathrm{P}}\right) 35 \%, 99 \%$ ee

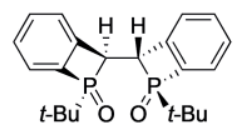

$\left(S_{p}, S_{p}\right) 21 \%, 98.8 \%$ ee Imamoto ref. [67d]

\section{Zhang ref. [67e]}

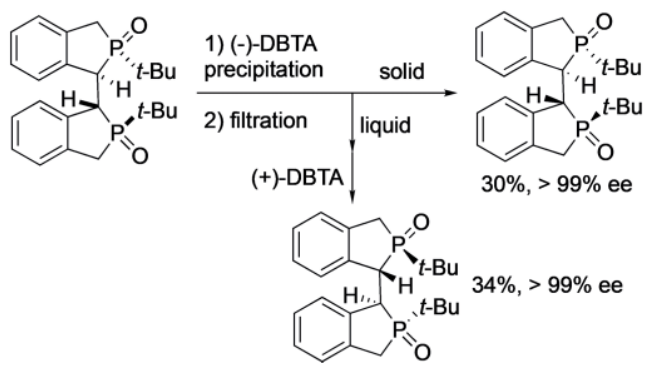

Scheme 26. Examples of resolution of diphosphine oxides via the complexation to DBTA.

These kinds of resolving agents could also be applied to the resolution of monophosphine oxides. In 2016, Bagi and Keglevich have summarized their studies on the formation of diastereomeric salts or molecular coordination complexes with
TADDOL derivatives or the calcium salts of tartaric acid derivatives for the resolution of various five- and six-membered Pheterocyclic phosphine oxides, phosphinates, a phosphine sulfide, and a phosphonic ester-amide. In general, the resolutions have been realized in excellent optical purities. (Scheme 27). ${ }^{666]}$ It is worth noting that, depending on the resolving agent used, both enantiomers of phospholene oxides could be obtained with a fine-tuning of the precipitation solvent mixture. There is evidence of the influence of the solvent dielectric constant on the selectivity and/or yield of the resolution. In some cases, the change from a protic solvent mixture to a non-protic one, one favored the precipitation of one enantiomer or the other one. Moreover, in terms of recognition, the nature of resolving agent is crucial for the host-guest complex formation. For example, one enantiomer of cyclic phosphinates can be recognized by (-)-TADDOL while the other enantiomer is better recognized by the (-)-spiro-TADDOL.

More recently, the resolution of acyclic monophosphine oxides has been studied by exploiting the molecular recognition ability of TADDOL or calcium salts of tartaric acid derivatives (Scheme 28). ${ }^{[69]}$ Protic solvents are not always necessary for a good complexation and precipitation of monophosphine oxides with spiro-TADDOL. Indeed, non-protic solvent mixtures, as toluene/heptane, could also be used. The resolutions have been realized with moderate to good yield (24-66\%) and high optical purities (89-99\%). Thanks to the XRD studies, ${ }^{[69 b]}$ they could clearly identify the secondary interaction responsible for the enantiomeric recognition, notably a strong $\mathrm{H}$-bridge ( $\mathrm{C}-\mathrm{O}-$ $\mathrm{H}$... O=P). Calcium tartaric acid salts could also form complexes with the ethyl-(2-methylphenyl)-phenylphosphine oxide. After

$$
\text { Resolving agents: }\left[\mathrm{Ca}^{2+},\right.
$$

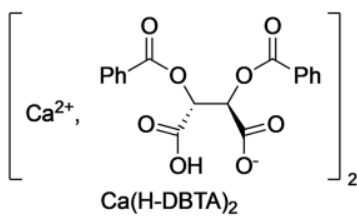

Resolutions of P-chiral compounds
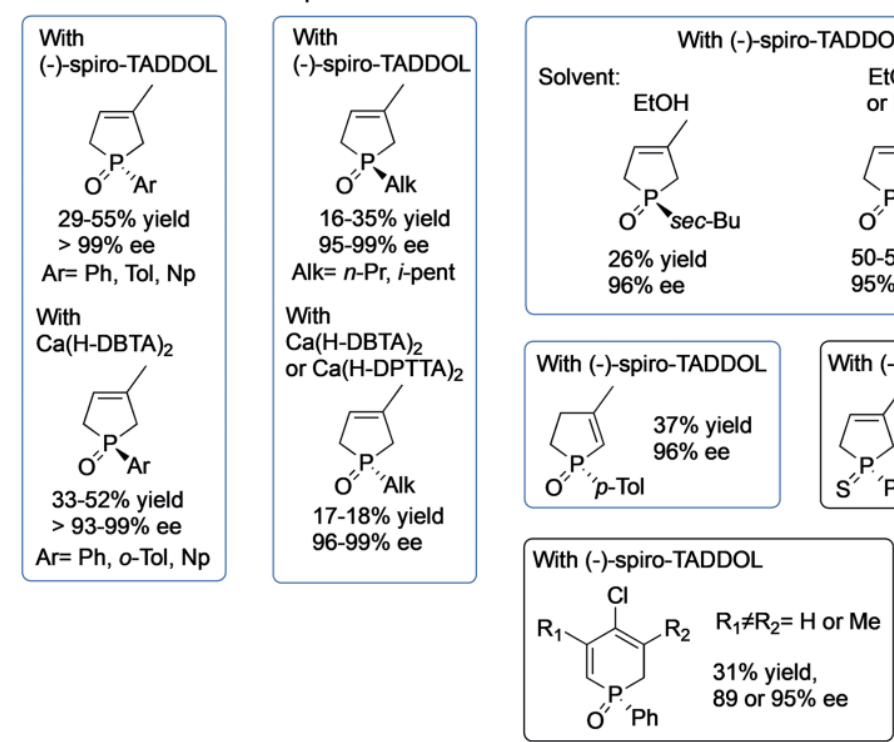

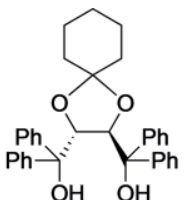

(-)-spiro-TADDOL

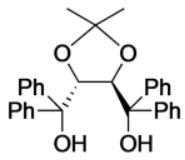

(-)-TADDOL

Scheme 27. Selected examples of resolution of five- and six-membered P-heterocyclic phosphorus compounds obtained in high enantiomeric excesses. 
washing and extraction, the enantiopure monophosphine oxide could be obtained in $47 \%$ yield and $99 \%$ ee. A fine-tuning of the solvent nature was again necessary to reach these optimum host-guest complexations.

$$
\begin{gathered}
\mathrm{R}_{1} \underset{(+/-)}{\stackrel{O}{P}-P h} \\
\mathrm{R}_{2}
\end{gathered}
$$

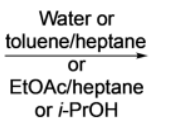

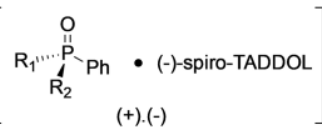

$\mathrm{R}_{1}=\mathrm{Me}$ or Et

$\mathrm{R}_{2}=$ Alk (Et, $n$ - or $i-\operatorname{Pr}, n$-pent, Cy, $t$-Bu)

or $o-$ Tol

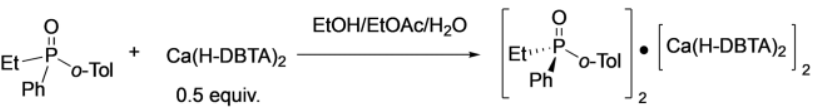

$$
\begin{aligned}
& \text { (+/-) }
\end{aligned}
$$

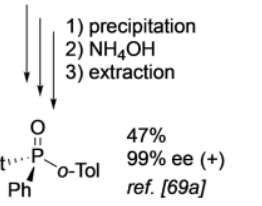

Scheme 28. Resolution of monophosphine oxides via the complexation to the spiro-TADDOL or $\mathrm{Ca}^{2+}$ - DBTA salt.

\section{Conclusion}

The introduction of the chiral element for the synthesis of $\mathrm{P}$ stereogenic molecules has acknowledged many developments in the past decades. Strategies based on the use of chiral auxiliaries attached to the phosphorus or on the use of chiral stoichiometric reagents have attracted most of the work and are still among the most general ones. Nevertheless, several catalytic enantioselective methods have recently emerged as powerful synthetic alternatives. However, these synthetic approaches still suffer from two important disadvantages:

a) the direct catalytic asymmetric synthesis of P-stereogenic P(III) compounds using transition-metal catalysts has been largely underdeveloped up to now, probably due to the compatibility issues between free phosphine-derived substrates/ products and transition-metals.

b) the organocatalyzed enantioselective synthesis of P-chiral molecules is to date an utterly underdeveloped field.

For these reasons, the synthesis of P-chiral compounds remains a challenging area of research, and one can foresee numerous developments in the near future, to meet the growing demand for chiral phosphorus molecules.
[1] D. E. C. Corbridge Phosphorus: Chemistry, Biochemistry and Technology, Sixth Edition, CRC Press, 2013.

[2] a) M. C. Allen, W. Fuhrer, B. Tuck, R. Wade, J. M. Wood, J. Med. Chem. 1989, 32, 1652-61; b) W. W. Smith, P. A. Bartlett, J. Am. Chem. Soc. 1998, 120, 4622-28.

[3] a) P. Kafarski, B. Lejczak, Curr. Med. Chem. Anti-Cancer Agents 2001, 1, $301-12$; b) for a review on $\alpha$-amino phosphorus oxides compounds in medicinal chemistry: A. Mucha, P. Kafarski, L. Berlicki, J. Med. Chem. 2011, 54, 5955-80.

[4] G. Forlani, L. Berlicki, M. Duo, G. Dziedziola, S. Gilberti, M. Bertazzini, P. Kafarski, J. Agric. Food Chem. 2013, 61, 6792-98.

[5] a) N. Long, X.-J. Cai, B.-A. Song, S. Yang, Z. Chen, P. S. Bhadury, D.-Y. Hu, L.-H. Jin, W. Xue, J. Agric. Food Chem. 2008, 56, 5242-46; b) M.-H. Chen, Z. Chen, B.-A. Song, P. S. Bhadury, S. Yang, X.-J. Cai, D.-Y. Hu, W. Xue, S. Zeng, J. Agric. Food Chem. 2009, 57, 1383-88. For a review: V. P. Kukhar, H. R. Hudson, Editors, Aminophosphonic and Aminophosphinic Acids: Chemistry and Biological Activity, Wiley, 2000.

[6] a) A. Börner, Editor (Eds.), Phosphorus Ligands in Asymmetric Catalysis; Synthesis and Applications, Wiley-VCH Verlag GmbH \& Co. KGaA, 2008; b) For a recent review on secondary phosphine oxides coordination, see: A. Gallen, A. Riera, X. Verdaguer, A. Grabulosa, Catal. Sci. Technol. 2019, 9, 5504-61.

[7] For a review on phosphine organocatalysis, see: a) H. Guo, Y. C. Fan, Z. Sun, Y. Wu, O. Kwon, Chem. Rev. 2018, 118, 10049-10293. For a review on phosphine oxides organocatalysis, see: b) T. Ayad, A. Gernet, J.-L. Pirat, D. Virieux, Tetrahedron 2019, 75, 4385-4418.

[8] For studies on the configurational stability of P-stereogenic phosphines: a) R. D. Baechler, K. Mislow, J. Am. Chem. Soc. 1970, 92, 3090-93; b) J. Holz, H. Jiao, M. Gandelman, A. Börner, Eur. J. Org. Chem. 2018, 2018, 2984-94.

[9] For selected recent examples, see: a) T. Imamoto, K. Sugita, K. Yoshida, J. Am. Chem. Soc. 2005, 127, 11934-35; b) M. P. Watson, E. N. Jacobsen, J. Am. Chem. Soc. 2008, 130, 12594-95; c) A. M. Taylor, R. A. Altman, S. L. Buchwald, J. Am. Chem. Soc. 2009, 131, 9900-01; d) Y. Shibata, K. Tanaka, J. Am. Chem. Soc. 2009, 131, 12552-53; e) M. Jin, L. Adak, M. Nakamura, J. Am. Chem. Soc. 2015, 137, 7128-34; f) W. You, M. K. Brown, J. Am. Chem. Soc. 2015, 137, 14578-81; g) E. Salomó, S. Orgué, A. Riera, X. Verdaguer, Angew. Chem. Int. Ed. 2016, 55, 7988-92; Angew. Chem. 2016, 128, 8120; h) Z. Gan, M. Zhi, R. Han, E.-Q. Li, Z. Duan, F. Mathey, Org. Lett. 2019, 21, 2782-85. For a review on P-stereogenic cyclic benzoxaphospholes in asymmetric catalysis, see: i) G. Xu, C. H. Senanayake, W. Tang, Acc. Chem. Res. 2019, 52, 1101-12.

[10] J. Meisenheimer, L. Lichtenstadt, Ber. Dtsch. Chem. Ges. 1911, 44, 35659.

[11] This topic has been covered in the following review articles: a) K. M. Pietrusiewicz, M. Zablocka, Chem. Rev. 1994, 94, 1375-411; b) A. Grabulosa, J. Granell, G. Muller, Coord. Chem. Rev. 2007, 251, 25-90; c) A. Grabulosa, Editor (Eds.), P-Stereogenic Ligands in Enantioselective Catalysis, RSC Publishing: Cambridge, 2011; d) M. Dutartre, J. Bayardon, S. Jugé, Chem. Soc. Rev. 2016, 45, 5771-94.

[12] W. B. Farnham, R. K. Murray, K. Mislow, J. Am. Chem. Soc. 1970, 92, 580910.

[13] a) L.-B. Han, C.-Q. Zhao, S. Onozawa, M. Goto, M. Tanaka, J. Am. Chem. Soc. 2002, 124, 3842-43; b) Q. Xu, C.-Q. Zhao, L.-B. Han, J. Am. Chem. Soc. 2008, 130, 12648-55; c) Y.-M. Sun, N. Xin, Z.-Y. Xu, L.-J. Liu, F.-J. Meng, H. Zhang, B.-C. Fu, Q.-J. Liang, H.-X. Zheng, L.-J. Sun, C.-Q. Zhao, L.-B. Han, Org. Biomol. Chem. 2014, 12, 9457-65; d) For an account: T. Chen, L.-B. Han, Synlett 2015, 26, 1153-1163.

[14] a) O. Berger, J.-L. Montchamp, Angew. Chem. Int. Ed. 2013, 52, 11377-80; Angew. Chem. 2013, 125, 11587; b) O. Berger, J.-L. Montchamp, Org. Biomol. Chem. 2016, 14, 7552-7562.

[15] a) A. Leyris, J. Bigeault, D. Nuel, L. Giordano, G. Buono, Tetrahedron Lett. 2007, 48, 5247-50; b) D. Moraleda, D. Gatineau, D. Martin, L. Giordano, G. Buono, Chem. Commun. 2008, 3031-33; c) D. Gatineau, L. Giordano, G. Buono, J. Am. Chem. Soc. 2011, 133, 10728-10731.

[16] A. Wlodarczyk, A. E. Koziol, M. Stankevic, Eur. J. Org. Chem. 2018, 2018, 1589-1600.

[17] a) S. Jugé, M. Stéphan, J. A. Laffitte, J. P. Genêt, Tetrahedron Lett. 1990, 31, 6357-60; b) S. Jugé, M. Stéphan, R. Merdès, J. P. Genêt, S. HalutDesportes, J. Chem. Soc., Chem. Commun. 1993, 531-533. 
[18] a) E. B. Kaloun, R. Merdès, J.-P. Genêt, J. Uziel, S. Jugé, J. Organomet. Chem. 1997, 529, 455-63; b) D. Moulin, S. Bago, C. Bauduin, C. Darcel, S. Jugé, Tetrahedron: Asymmetry 2000, 11, 3939-3956; c) E. A. Colby, T. F. Jamison, J. Org. Chem. 2003, 68, 156-166; d) C. Bauduin, D. Moulin, E. B. Kaloun, C. Darsel, S. Jugé, J. Org. Chem. 2003, 68, 4293-4301; e) J. Bayardon, Y. Rousselin, S. Jugé, Org. Lett. 2016, 18, 2930-2933; f) C. F. Czauderna, A. M. Z. Slawin, D. B. Cordes, J. I. van der Vlugt, P. C. J. Kamer, Tetrahedron 2019, 75, 47-56.

[19] a) T. León, A. Riera, X. Verdaguer, J. Am. Chem. Soc. 2011, 133, 5740-43; b) H. Zijlstra, T. León, A. de Cozar, C. Fonseca Guerra, D. Byrom, A. Riera, X. Verdaguer, F. M. Bickelhaupt, J. Am. Chem. Soc. 2013, 135, 4483-4491.

[20] a) S. Orgué, A. Flores-Gaspar, M. Biosca, O. Pamies, M. Dieguez, A. Riera, X. Verdaguer, Chem. Commun. 2015, 51, 17548-51; b) A. Prades, S. NuñezPertiñez, A. Riera, X. Verdaguer, Chem. Commun. 2017, 53, 4605; c) E. Salomo, A. Prades, A. Riera, X. Verdaguer, J. Org. Chem. 2017, 82, 70657069.

[21] E. Salomo, S. Orgué, A. Riera, X. Verdaguer, Synthesis 2016, 48, 26592663.

[22] J. V. Carey, M. D. Barker, J. M. Brown, M. J. H. Russell, J. Chem. Soc., Perkin Trans. 1 1993, 831-839.

[23] A. Leyris, D. Nuel, L. Giordano, M. Achard, G. Buono, Tetrahedron Lett. 2005, 46, 8677-8680.

[24] a) Z. S. Han, N. Goyal, M. A. Herbage, J. D. Sieber, B. Qu, Y. Xu, Z. Li, J. T. Reeves, J.-N. Desrosiers, S. Ma, N. Grinberg, H. Lee, H. P. R. Mangunuru, Y. Zhang, D. Krihnamurthy, B. Z. Lu, J. J. Song, G. Wang, C. H. Senanayake, J. Am. Chem. Soc. 2013, 135, 2474-77; b) Z. S. Han, L. Zhang, Y. Xu, J. D. Sieber, M. A. Marsini, Z. Li, J. T. Reeves, K. R. Fandrick, N. D. Patel, J.-N. Desreosiers, B. Qu, A. Chen, D. M. Rudzinski, L. P. Samankumara, S. Ma, N. Grinberg, F. Roschangar, N. K. Yee, G. Wang, J. J. Song, C. H. Senanayake, Angew. Chem. Int. Ed. 2015, 54, 5474-5477; Angew. Chem. 2015, 127, 5564-5567.

[25] a) L. Copey, L. Jean-Gérard, E. Framery, G. Pilet, V. Robert, B. Andrioletti, Chem. Eur. J. 2015, 21, 9057-9061; b) A. D'onofrio, L. Copey, L. JeanGérard, C. Goux-Henry, G. Pilet, B. Andrioletti, E. Framery, Org. Biomol. Chem. 2015, 13, 9029-34.

[26] M. A. del Aguila-Sanchez, Y. Navarro, J. Garcia Lopez, G. P. Guedes, F. Lopez Ortiz, Dalton Trans. 2016, 45, 2008-22.

[27] a) E. Bergin, C. T. O'Connor, S. B. Robinson, E. M. McGarrigle, C. P. O'Mahony, D. G. Gilheany, J. Am. Chem. Soc. 2007, 129, 9566-67; b) K. V. Rajendran, D. G. Gilheany, Chem. Commun. 2012, 48, 10040-42; c) K. Nikitin, K. V. Rajendran, H. Muller-Bunz, D. G. Gilheany, Angew. Chem. Int. Ed. 2014, 53, 1906-09; Angew. Chem. 2014, 126, 1937; d) K. V. Rajendran, K. Nikitin, D. G. Gilheany, J. Am. Chem. Soc. 2015, 137, 9375-81.

[28] For a mechanistic study, see: E. V. Jennings, K. Nikitin, Y. Ortin, D. G. Gilheany, J. Am. Chem. Soc. 2014, 136, 16217-26.

[29] The stereospecific reduction of phosphine oxides to phosphine-boranes using $\left[\mathrm{R}_{3} \mathrm{O}\right] \mathrm{BF}_{4}$ or MeOTf as electrophiles in combination with $\mathrm{NaBH}_{4}$ has also been described: K. V. Rajendran, D. G. Gilheany, Chem. Commun. 2012, 48, 817-19.

[30] A. R. Muci, K. R. Campos, D. A. Evans, J. Am. Chem. Soc. 1995, 117, 907576.

[31] T. Imamoto, J. Watanabe, Y. Wada, H. Masuda, H. Yamada, H. Tsuruta, S. Matsukawa, K. Yamaguchi, J. Am. Chem. Soc. 1998, 120, 1635-36.

[32] a) Y. Yamanoi, T. Imamoto, J. Org. Chem. 1999, 64, 2988-89; b) K. Nagata, S. Matsukawa, T. Imamoto, J. Org. Chem. 2000, 65, 4185-88; c) M. J. Johansson, L. O. Schwartz, M. Amedjkouh, N. C. Kann, Eur. J. Org. Chem. 2004, 2004, 1894-96; d) M. J. Johansson, L. Schwartz, M. Amedjkouh, N. C. Kann, Tetrahedron: Asymmetry 2004, 15, 3531-38; e) C. Genet, S. J. Canipa, P. O'Brien, S. Taylor, J. Am. Chem. Soc. 2006, 128, 9336-37; f) F. Dolhem, M. J. Johansson, T. Antonsson, N. Kann, J. Comb. Chem. 2007, 9, 477-86; g) J. Granander, F. Secci, S. J. Canipa, P. O'Brien, B. Kelly, J. Org. Chem. 2011, 76, 4794-99.

[33] a) J. J. Gammon, S. J. Canipa, P. O'Brien, B. Kelly, S. Taylor, Chem. Commun. 2008, 3750-52; b) J. J. Gammon, V. H. Gessner, G. R. Barker, J. Granander, A. C. Whitwood, C. Strohmann, P. O'Brien, B. Kelly, J. Am. Chem. Soc. 2010, $132,13922-27$.

[34] C. Popovici, P. Oña-Burgos, I. Fernàndez, L. Roces, S. García-Granda, M. J. Iglesias, F. López Ortiz, Org. Lett. 2010, 12, 428-31.

[35] B. Wolfe, T. Livinghouse, J. Am. Chem. Soc. 1998, 120, 5116-17.
[36] a) J. Holt, A. M. Maj, E. P. Schudde, K. M. Pietrusiewicz, L. Sieron, W. Wieczorek, T. Jerphagnon, I. W. C. E. Arends, U. Hanefeld, A. J. Minnaard, Synthesis 2009, 2061-65; b) F. A. Kortmann, M.-C. Chang, E. Otten, E. P. A. Couzijn, M. Lutz, A. J. Minnaard, Chem. Sci. 2014, 5, 1322-27.

[37] This topic has been partially covered in the following review articles: a) D. S. Glueck, Synlett 2007, 17, 2627-34; b) J. S. Harvey, V. Gouverneur, Chem. Commun. 2010, 46, 7477-85.

[38] a) T. Oshiki, T. Imamoto, J. Am. Chem. Soc. 1992, 114, 3975-77; b) T. Imamoto, T. Oshiki, T. Onozawa, M. Matsuo, T. Hikosaka, M. Yanagawa, Heteroat. Chem. 1992, 3, 563-75.

[39] M. Al-Masum, G. Kumaraswamy, T. Livinghouse, J. Org. Chem. 2000, 65, 4776-78.

[40] J. R. Moncarz, T. J. Brunker, D. S. Glueck, R. D. Sommer, A. L. Rheingold, J. Am. Chem. Soc. 2003, 125, 1180-81.

[41] a) S. Pican, A.-C. Gaumont, Chem. Commun. 2005, 2393-95; b) B. Join, D. Mimeau, O. Delacroix, A.-C. Gaumont, Chem. Commun. 2006, 3249-51.

[42] For studies on the pyramidal inversion of metal phosphido complexes, see: a) J. M. Rogers, T. P. S. Wagner, D. S. Marynick, Inorg. Chem. 1994, 33, 3104-10; b) S. K. Gibbons, C. R. D. Valleau, J. L. Peltier, M. F. Cain, R. P. Hughes, D. S. Glueck, J. A. Golen, A. L. Rheingold, Inorg. Chem. 2019, 58, 8854-65.

[43] a) J. R. Moncarz, N. F. Laritcheva, D. S. Glueck, J. Am. Chem. Soc. 2002, 124, 13356-57; b) N. F. Blank, J. R. Moncarz, T. J. Brunker, C. Scriban, B. J. Anderson, O. Amir, D. S. Glueck, L. N. Zakharov, J. A. Golen, C. D. Incarvito, A. L. Rheingold, J. Am. Chem. Soc. 2007, 129, 6847-58.

[44] V. S. Chan, R. G. Bergman, F. D. Toste, J. Am. Chem. Soc. 2007, 129, 15122 23.

[45] C. Scriban, D. S. Glueck, J. Am. Chem. Soc. 2006, 128, 2788-89.

[46] a) V. S. Chan, I. C. Stewart, R. G. Bergman, F. D. Toste, J. Am. Chem. Soc. 2006, 128, 2786-87; b) V. S. Chan, M. Chiu, R. G. Bergman, F. D. Toste, J. Am. Chem. Soc. 2009, 131, 6021-32.

[47] C. Scriban, I. Kovacik, D. S. Glueck, Organometallics 2005, 24, 4871-74.

[48] Y. Huang, Y. Li, P.-H. Leung, T. Hayashi, J. Am. Chem. Soc. 2014, 136, 4865 68.

[49] R. Beaud, R. J. Phipps, M. J. Gaunt, J. Am. Chem. Soc. 2016, 138, 1318386.

[50] Y. Zhang, H. He, Q. Wang, Q. Cai, Tetrahedron Lett. 2016, 57, 5308-11.

[51] Q. Dai, W. Li, Z. Li, J. Zhang, J. Am. Chem. Soc. 2019, 141, 20556-64.

[52] X.-T. Liu, Y.-Q. Zhang, X.-Y. Han, S.-P. Sun, Q.-W. Zhang, J. Am. Chem. Soc. 2019, 141, 16584-89.

[53] a) G. Nishida, K. Noguchi, M. Hirano, K. Tanaka, Angew. Chem. Int. Ed. 2008, 47, 3410-13; Angew. Chem. 2008, 120, 3458; b) R.-Y. Zhu, L. Chen, X. S. Hu, F. Zhouand, J. Zhou, Chem. Sci. 2020, 11, 97-106.

[54] Y. Zheng, L. Guo, W. Zi, Org. Lett. 2018, 20, 7039-43.

[55] Y. Zhang, F. Zhang, L. Chen, J. Xu, X. Liu, X. Feng, ACS Catal. 2019, 9, 4834-40.

[56] J. S. Harvey, S. J. Malcolmson, K. S. Dunne, S. J. Meek, A. L. Thompson, R. R. Schrock, A. H. Hoveyda, V. Gouverneur, Angew. Chem. Int. Ed. 2009, 48, 762-66; Angew. Chem. 2009, 121, 776.

[57] Z. Wang, T. Hayashi, Angew. Chem. Int. Ed. 2018, 57, 1702-06; Angew. Chem. 2018, 130, 1718.

[58] H. Fernández-Pérez, A. Vidal-Ferran, Org. Lett. 2019, 21, 7019-23.

[59] Pd(0)]: a) Z.-Q. Lin, W.-Z. Wang, S.-B. Yan, W.-L. Duan, Angew. Chem. Int. Ed. 2015, 54, 6265-6269; Angew. Chem. 2015, 127, 6363; b) G. Xu, M. Li, S. Wang, W. Tang, Org. Chem. Front. 2015, 2, 1342-1345; [Pd(II)]: c) Z.-J. Du, J. Guan, G.-J. Wu, P. Xu, L.-X. Gao, F.-S. Han, J. Am. Chem. Soc. 2015, 137, 632-35. [Rh(III)]: d) Y. Sun, N. Cramer, Angew. Chem. Int. Ed. 2017, 56, 364-67; Angew. Chem. 2017, 129, 370-73; e) Y. Sun, N. Cramer, Chem. Sci. 2018, 9, 2981-85. [Ir(III)]: f) Y.-S. Jang, M. Dieckmann, N. Cramer, Angew. Chem. Int. Ed. 2017, 56, 15088-92; Angew. Chem. 2017, 129, 15284; g) Y.-S. Jang, L. Wozniak, J. Pedroni, N. Cramer, Angew. Chem. Int. Ed. 2018, 57, 12901-5; Angew. Chem. 2018, 130, 13083. For a recent review on the synthesis of heteroatom-centered chiral molecules through $\mathrm{C}-\mathrm{H}$ functionalization, see: h) J. Diesel, N. Cramer, ACS Catal. 2019, 9, 9164-77.

[60] H. Lebel, S. Morin, V. Paquet, Org. Lett. 2003, 5, 2347-49.

[61] a) Z. Huang, X. Huang, B. Li, C. Mou, S. Yang, B.-A. Song, Y. R. Chi, J. Am. Chem. Soc. 2016, 138, 7524-27; b) G.-H. Yang, Y. Li, X. Li, J.-P. Cheng, Chem. Sci. 2019, 10, 4322-27. 
[62] a) D. Hérault, G. Buono, N. Vanthuyne, C. Roussel, L. Giordano, D. Moraleda, D. Gatineau, J. Leclaire, D. Nuel, PCT Int. Appl. 2014, WO2014108487 A1 20140717; b) D. Gatineau, D. H. Nguyen, D. Hérault, N. Vanthuyne, J. Leclaire, L. Giordano, G. Buono, J. Org. Chem. 2015, 80, 4132-41.

[63] a) S. Lemouzy, D. H. Nguyen, V. Camy, M. Jean, D. Gatineau, L. Giordano, J.-V. Naubron, N. Vanthuyne, D. Hérault, G. Buono, Chem. Eur. J. 2015, 21 , 15607-21; b) S. Lemouzy, D. H. Nguyen, D. Gatineau, L. Giordano, D. Hérault, G. Buono, Pure Appl. Chem. 2016, 88, 333-39; c) For another example of borane-mediated stereospecific reduction of hydroxyalkylphosphine oxides, see: S. Sowa, M. Stankevic, A. Szmigielska, H. Maluszynska, A. E. Koziol, K. M. Pietrusiewicz, J. Org. Chem. 2015, 80, 1672-88.

[64] S. Lemouzy, M. Jean, L. Giordano, D. Hérault, G. Buono, Org. Lett. 2016, $18,140-43$.

[65] a) S. Lemouzy, R. Membrat, E. Olivieri, M. Jean, M. Albalat, D. Nuel, L. Giordano, D. Hérault, G. Buono, J. Org. Chem. 2019, 84, 4551-57. For a recent account on amino-phosphine: b) A. Cabré, A. Riera, X. Verdaguer, Acc. Chem. Res. 2020, 53, 676-89.
[66] a) The resolution methods via formation of diastereomeric phosphonium salts or metal complexes are documented in ref. ${ }^{[1 \mathrm{a}]}$ and ; b) P. Bagi, V. Ujj, M. Czugler, E. Fogassy, G. Keglevich, Dalton Trans. 2016, 45, 182342.

[67] a) Y. Hamada, F. Matsuura, M. Oku, K. Hatano, T. Shioiri, Tetrahedron Lett 1997, 38, 8961-64; b) T. Miura, T. Imamoto, Tetrahedron Lett. 1999, 40, 4833-36; c) J. Omelańczuk, A. Karaçar, M. Freytag, P. G. Jones, R. Bartsch, M. Mikołajczyk, R. Schmutzler, Inorg. Chim. Acta 2003, 350, 583-91; d) T. Imamoto, K. V. L. Crépy, K. Katagiri, Tetrahedron: Asymmetry 2004, 15, 2213-18; e) D. Liu, X. Zhang, Eur. J. Org. Chem. 2005, 2005, 646-49.

[68] For a review on phosphine oxides reduction, see D. Hérault, D. H. Nguyen, D. Nuel, G. Buono, Chem. Soc. Rev. 2015, 44, 2508-28.

[69] a) P. Bagi, B. Varga, A. Szilágyi, K. Karaghiosoff, M. Czugler, E. Fogassy, G. Keglevich, Chirality 2018, 30, 509-22; b) B. Varga, R. Herbay, G. Székely, T. Holczbauer, J. Madarász, B. Mátravölgyi, E. Fogassy, G. Keglevich, P. Bagi, Eur. J. Org. Chem. 2020, 2020, 1840-52. 